\title{
RILIEVO MAGNETICO DELLA SICILIA CENTRO-SETTENTRIONALE ${ }^{*}$ )
}

\author{
M. Giongi - E. Medi - F. Molina
}

Premessa. - Nel quadro di un rilevamento geofisico generale della Sicilia, predisposto dall'Istituto Naz. di Geofisica, è stato iniziato il lavoro di rilevamento magnetico del secondo ordine di una vasta zona nella parte centro-settentrionale dell'isola.

Il piano per l'esecuzione di una rete greofisica, programmato dall'I.N.G. per l'intero territorio nazionale $(1)$ e cominciato ad attuare in Sicilia, ha lo scopo di individuare e studiare le caratteristiche geofisiche regionali in rapporto con la struttura geologica superficiale e profonda e la tettonica del suolo nazionale.

La realizzazione di un simile piano costituirebbe un risultato di notevole importanza scientifica e pratica; si verreblero a possedere infatti i dati fondamentali per l'esame preliminare di quelle regioni da sottoporre eventualmente a ricerche specifiche di dettaglio quando ne risultasse un immediato interesse minerario.

Il territorio rilevato, come può vedersi dalle annesse carte delle isorlinamiche e delle isoanomale, è compreso tra S. Stefano di Canastra, Mistretta, Nicosia, S. Caterina, Caltanissetta, Alia. Ciminna, Termini Imerese.

La scelta di questa parte della Sicilia per l'inizio delle operazioni di campagna è stata motivata dal fatto che essendo in costruzione a Gihilmanna, presso Cefalù, un Osservatorio greofisico che comprende anche i padiglioni magnetici, si rendeva opportuno, oltre ad un rilicro di dettaglio locale nella zona dell'Osservatorio, anche un esame del comportamento regionale della più vasta zona circostante,

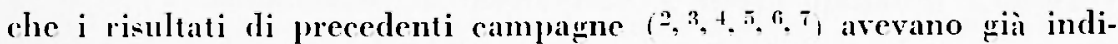
calo come pressoché " normale " dal punto di vista magnetico.

Prima della esecuzione pratica del rilievo è stato elaborato un dettagliato programma di lavoro, fissando una rete di stazioni di misure relative, convenientemente distribuite, delle componenti $H$ e $Z$ ad una distanza media di $5 \mathrm{~km}$, lungo circuiti stabiliti nella maniera

(*) Comunicazione presentata alla "Association Internationale de Magnétisme et electuicite terrestre " nella X Assemblea Generale dell'L.G.G.I. R Roma 1954. 
più opportuna e intersecantisi, compatiljilmente con la rete stradale della regione, in modo da ottenere stazioni ripetute di controllo oltre alla stazione base.

11 programma prestabilito, per necessita pratiche di lavoro, è stato poi in qualche parte modificato nel corso dell'esecuzione delle operazioni di rileramento; comunque l'esperienza acquisita in analoghi larori precedenti ci ha consentito di ridure al minimo gli imprevisti e di procedere con grande speditezza.

La zona rilevata copre un'area di $3.000 \mathrm{~km}^{2}$ ca.

Sono stati compiuti n. 9 circuiti comprendenti 124 stazioni complessive escludendo le stazioni ripetute.

Sono state battute 13 stazioni assolute dell'Istituto Geografico Militare tra cui una fondamentale.

Le operazioni di misure relative si sono svolte nei griorni dal 28 feb])raio al 10 marzo 1952.

Nel mese di marzo 1954 sono state poi eseguite alcune misure assolute allo scopo di ancorarvi la rete del ll ordine.

In questa nota vengono riportati e discussi i risultati delle misure magnetiche assolute e relative. $\mathrm{Ci}$ proponiamo di esporre in una nota successiva una elaborazione ulteriore dei dati stessi in rapporto con altri dati geofisici e con le attuali conoscenze weologiche.

Equipaggiamento strumentale per le misure relative ed assolute. Per la esecuzione della prima campagna di misure relative delle componenti $H$ e $Z$ sono state utilizzate:

a) 4 bilance tipo Schmidt di costruzione "Askania " di cui 2 con testa di registrazione a cellula fotoelettrica;

b) un apparato di registrazione a due tracee;

c) una terna di variografi per le componenti $D, H, Z$ tipo "Ruska » installato presso l'Osservatorio di Giljilmanna;

d) apparati di taratura con bobina di Felmholtz per le bilance "Askania " ed i variometri "Ruska ».

Per la esecuzione della campagna di misure assolute, in cui sono stati determinati gli elementi $D, H$ e $I$, sono state utilizzate invece le seguenti apparecchiature:

a) un teodolite da campo “Askania»; 
b) un induttore terrestre per misure di campagna "Askania »; c) 2 magnetometri a quarzo tipo La Cour (HTM) dell'، Askania $)$;

d) 2 bilance magnetiche per le componenti $H$ e $Z$ (per il controllo delle differenze $\Delta / l$ e $\Delta Z$ tra una stazione e l'altra);

e) un'apparecchiatura per la determinazione esatta del tempo comprendente: 2 cronometri tipo "Nardin », un cronografo registratore "Favag", un apparechio radio ricevente ad alimentazione autononla da campo.

Prescindendo dalle determinazioni delle costanti strumentali eseguite periodicamente durante le operazioni di campagna delle quali si parla più sotto, le caratteristiche generali strumentali delle 4 bilance di Schmidt erano quelle della tabella $n$. 1 .

tal). n. 1

\begin{tabular}{|c|c|c|c|c|c|}
\hline Equipaggio & Comp. & $\begin{array}{l}\text { Campo inedio } \\
\text { di taratura }\end{array}$ & $\begin{array}{l}\text { Costante di } \\
\text { scala : } \varepsilon\end{array}$ & $\begin{array}{l}\text { Coeff. di temp. } \\
\text { fra } 10^{\circ} \text { e } 45^{\circ}\end{array}$ & $\begin{array}{l}\text { Errore m. } \\
\text { per } 5 \text { letture }\end{array}$ \\
\hline $\begin{array}{l}\text { n. } 402-3 \\
\text { bil. n. } 509998\end{array}$ & Z & $\mathrm{Z}=0,3500 \mathrm{I}$ & $10,0 \pm 0,16 \dddot{u}$ & 0,0 & $\pm 0,6 \gamma$ \\
\hline $\begin{array}{l}\text { n. } 291-2 \\
\text { bil. n. } 509999\end{array}$ & $\mathrm{H}$ & $\begin{array}{l}H=0,2510 \\
Z=0,3500\end{array}$ & $10,0 \pm 0,10 "$ & 0,0 & $\pm 0,5 \gamma$ \\
\hline $\begin{array}{l}\text { n. } 374-5 \\
\text { bil. n. } 509993\end{array}$ & Z (Reg.) & $\mathrm{Z}=0,3500$ & $10,0 \pm 0,16 n$ & 0,0 & $\pm 0,5 \gamma$ \\
\hline $\begin{array}{l}\text { n. } 410-11 \\
\text { bil. n. } 509966\end{array}$ & H (Reg.) & $\begin{array}{l}\mathrm{Z}=0,3500 \\
\mathrm{H}=0,2510\end{array}$ & $10,0 \div 0,10 "$ & 0,0 & $\pm 0,5 \gamma$ \\
\hline
\end{tabular}

Come si vede nella detta tabella le intensità dei campi di taratura degli equipaggi erano tali che, dati i limiti di variabilità dei campi osservati durante la campagna, non è stato mai necessario adoperare i magneti ausiliari.

Come si vedrì nel seguito, per la esiguità delle anomalie da porre in rilievo, questa ì stata una circostanza favorevole, in quanto ha permesso di evitare la introduzione di possibili cause nii errore connesse con la correzione delle misure per i magneti ausiliari.

Le costanti strumentali dei variometri a filo di quarzo HTM de- 
terminate presso l'Osservatorio Magnetico di Fürstenfeldbruek (Germania) sono indicate nella tabella $n .2$.

tab. n. 2

\begin{tabular}{|c|c|c|c|c|c|c|}
\hline $\begin{array}{l}\text { Magneto- } \\
\text { metro }\end{array}$ & $\begin{array}{c}\text { Costante } \\
\text { C }\end{array}$ & $\begin{array}{c}\text { Cost. di } \\
\text { tcmp. fra } \\
5^{\circ} e^{3} 35^{\circ} \mathrm{C} \text {. } \\
\mathrm{C}^{\prime}\end{array}$ & $\begin{array}{l}\text { Angrolo } \\
\text { di rotiz. } \\
\text { dello } \\
\text { strum. }\end{array}$ & $\begin{array}{l}\text { Torsione } \\
\text { residuil }\end{array}$ & $\begin{array}{l}\text { Cocff. di } \\
\text { induz. fino } \\
\text { a } J \mathrm{H}= \\
:=6+00 \because\end{array}$ & $\begin{array}{l}\text { Errore m. } \\
\text { di } 3 \text { letture } \\
\text { complete }\end{array}$ \\
\hline 1.. 5010167 & 1.25674 & $1,287.10^{-4}$ & $6 \pi$ & $16^{\prime}, 5$ & 0 & $\pm 1,5 Y$ \\
\hline 11. 5010168 & 1.20267 & $0,794.10^{-4}$ & $+\pi$ & $3^{\prime}, 6$ & 0 & $\pm 1,5 \because$ \\
\hline
\end{tabular}

Come ì noto dalla teoria del variometro a torsione di La Cour (s) il campo $H$ si ottiene mediante la relazione:

$$
\log H=C+C^{\prime} \Delta t-K H c \text { s } \varphi-l \log \operatorname{sen} ?
$$

dove:

$$
\varphi=\frac{1}{2}\left[\operatorname{sen}\left(a_{1}+\alpha\right)+\operatorname{sen}\left(a_{2}-\alpha\right)\right]
$$

e $a_{1}$ ì l'angolo di deviazione del magnete per una rolazione dello strumento di $2 \mathrm{k}-$ in un verso, $a:$ i l'angolo di deviazione del magnete per una analoga rotazione dello strumento in verso opposto e finalmente:

$$
\operatorname{tg} \alpha=\frac{\operatorname{sen} a_{1}-\operatorname{sen} a_{n}}{2-\left(\cos a_{1}+\cos a_{n}\right)}
$$

Sono stati usati 2 magnetometri HTM per avere una possibilità di confronto; per quanto ci consta $\dot{e}$ la prima volta che vengono usati in Italia detti magnetometri che, ben tarati e controllati, consentono una misura di $H$ molto spedita c precisa; infalti le determinazioni di $H$ eseguite con uno stesso strumento, fatte le debite coriezioni per la variazione diurna tra una determinazione e l'altra, concordano in genere entro 1 o $2 \%$. 
Operazioni di campagna per le misure assolute e relative.

a) Misure relative. - La campagna di misure relative si è svolta, come detto sopra, dal 28 febbraio al 10 marzo 1952.

Le prime operazioni sono state quelle di taratura dei variografi "Ruska ) dell'Osservatorio magnetico di Gibilmanna, la scelta della stazione base, l'impianto della stazione di registrazione ausiliaria da campo e la taratura dei variometri.

La stazione base è stata scelta per ragioni di opportunita di lavoro in una zona di facile accesso, magneticamente normale, nel punto di coordinate:

$$
\varphi=38^{\circ} 01^{\prime} 32^{\prime \prime} N \quad \lambda=1^{\circ} 34^{\prime} 35^{\prime \prime} E \text { (Monte Mario) }
$$

a $80 \mathrm{~m}$. dalla strada Cefalù-Gibilmanna a circa $2 \mathrm{Km}$. da Cefalù ; in essa hanno avuto inizio e termine le misure di tutti i circuiti.

In ogni stazione le misure sono state eseguite in almeno tre punti alla distanza di circa $20 \mathrm{~m}$. l'uno dall'altro onde controllare l'assenza di perturbazioni accidentali. In ogni punto venivano eseguite, sia per la bilancia verticale che per la orizzontale, quattro misure. Il valore medio delle misure $i$ stato attribuito al baricentro dei punti.

I dati topografici e magnetici delle singole stazioni sono contenuti nelle tabelle n. 3, 4, 5. (L'asterisco indica le misure ripetute).

Le coordinate geografiche delle stazioni riportate nella tabella n. 3 sono state misurate su carte topografiche alla scala $1: 25.000$.

Naturalmente è stata posta ogrni cura nella scelta delle stazioni di osservazione, sia durante la redazione del programma generale del rilevamento, sia durante la esecuzione pratica delle operazioni di campagna, allo scopo di evitare ogni causa di perturbazione naturale o artificiale presente (discontinuità naturali, acquedotti, linee elettriche, ferrovie, strade, industrie, case ecc.) e, per quanto prevedibile, anche futura (eventuale sviluppo di attività umane perturbatrici), onde rendere possibili eventuali ripetizioni delle stazioni stesse.

Nelle stazioni ritenute di una certa importanza sono stati piantati picchetti di legno.

Sono state prese sempre, per quanto possibile, opportune precauzioni nella scelta dei punti di stazione allo scopo di ridurre gli effetti sia della insolazione diretta sullo strumento sia del vento, piazzando gli strumenti in posti possibilmente riparati e non si è mai ravvisata, durante la campagna, la necessità di porli al riparo della tenda amagnetica di cui le squadre erano fornite. 
TABELLA $n .3$.

\begin{tabular}{|c|c|c|c|c|c|c|}
\hline Circuito & St. & $\varphi \mathrm{N}$ & $\begin{array}{c}\lambda \mathrm{E} \\
\text { (M. Mario) }\end{array}$ & $\mathrm{l} \mathrm{m}$ & $\begin{array}{c}\text { altitudine } \\
\mathrm{m}\end{array}$ & $\begin{array}{l}\text { Temp. } \\
\text { in } C^{0}\end{array}$ \\
\hline \multirow[t]{2}{*}{ I } & $1(\mathrm{~B})$ & $38^{\circ} 01^{\prime} 32^{\prime \prime}$ & $1.34^{\prime} 35^{\prime \prime}$ & 842 & 160 & 13,9 \\
\hline & $\begin{array}{c}1 \\
2\end{array}$ & $38^{\circ} 01^{\prime} 14^{\prime \prime}$ & $1 \circ 34^{\prime} 58^{\prime \prime}$ & 9) 27 & 370 & 13,9 \\
\hline \multirow[t]{8}{*}{ 1-III-52 } & 3 & $38^{\circ} 00^{\prime} 50^{\prime \prime}$ & $1^{\circ} 34^{\prime} 13^{\prime \prime}$ & 955 & 550 & 13,7 \\
\hline & 4 & $37^{\circ} 59^{\prime} 23^{\prime \prime}$ & $1^{\circ} 33^{\prime} 45^{\prime \prime}$ & 1016 & 700 & 13,5 \\
\hline & 5 & $37059^{\prime} 12^{\prime \prime}$ & $1 \cdot 34^{\prime} 10^{\prime \prime}$ & 1100 & 875 & 11,9 \\
\hline & 6 & $37^{\circ} 59^{\prime} 13^{\prime \prime}$ & $1^{\circ} 34^{\prime} 21^{\prime \prime}$ & 1125 & 975 & 12,0 \\
\hline & 7 & $37^{\circ} 59^{\prime} 22^{\prime \prime}$ & $1^{\circ} 34^{\prime} 28^{\prime \prime}$ & 1147 & 980 & 10,0 \\
\hline & 8 & $37^{\circ} 59^{\prime} 18^{\prime \prime}$ & $1 \circ 3 t^{\prime} 24^{\prime \prime}$ & 1200 & 1005 & 9,7 \\
\hline & 9 & $37^{\circ} 59^{\prime} 25^{\prime \prime}$ & $103 t^{\prime} 16^{\prime \prime}$ & 1400 & 950 & 9,5 \\
\hline & $\bar{B}$ & $38^{\circ} 01^{\prime} 32^{*}$ & $1^{\circ} 34^{\prime} 35^{\prime \prime}$ & 1612 & 160 & 9,5 \\
\hline \multirow[t]{2}{*}{ II } & $\mathrm{B}$ & $38^{\circ} 01^{\prime} 32^{\prime \prime}$ & $1^{\circ} 34^{\prime} 35^{\prime \prime}$ & 1612 & 160 & 9,5 \\
\hline & 10 & $38^{\circ} 01^{\prime} 28^{\prime \prime}$ & $1^{\circ} 30^{\prime}+4^{\prime \prime}$ & 1646 & 45. & 13,5 \\
\hline \multirow[t]{6}{*}{ 1-III-52 } & 11 & $38^{\circ} 00^{\prime} 19^{\prime \prime}$ & $1.28^{\prime} 57^{\prime \prime}$ & 1707 & 20 & 12,6 \\
\hline & 12 & $37^{\circ} 59^{\prime} 15^{\prime \prime}$ & $1^{\circ} 29^{\prime} 18^{\prime \prime}$ & 1725 & 170 & 15,0 \\
\hline & 13 & $37^{\circ} 59^{\prime} 00^{\prime \prime}$ & $1 \circ 29^{\prime} 35^{\prime \prime}$ & 1743 & 250 & 15,3 \\
\hline & 14 & $37^{\circ} 58^{\prime} 39^{\prime \prime}$ & $1.3 l^{\prime} 38^{\prime \prime}$ & 1801 & 600 & 15,5 \\
\hline & 15 & $37^{\circ} 58^{\circ} 05^{\prime \prime}$ & $1^{\circ} 31^{\prime} 18^{\prime \prime}$ & 1825 & 700 & 15,0 \\
\hline & $B$ & $38^{\circ} 01^{\prime} 32^{\prime \prime}$ & $1^{\circ} 34^{\prime} 35^{\prime \prime}$ & 1914 & 160 & 14,8 \\
\hline \multirow[t]{2}{*}{ III } & $B$ & $38^{\circ} 01^{\prime} 32^{\prime \prime}$ & $1^{\circ} 34^{\prime} 35^{\prime \prime}$ & 937 & 160 & 14,7 \\
\hline & 16 & $38^{\circ} 01^{\prime} 37^{\prime \prime}$ & $1^{\circ} 32^{\prime} 37^{\prime \prime}$ & 1000 & 50 & 15,5 \\
\hline \multirow[t]{2}{*}{$2-I I I-52$} & 10 & $38^{\circ} 01^{\prime} 28^{\prime \prime}$ & $1^{\circ} 30^{\prime} 44^{\prime \prime}$ & 1013 & 45 & 15,7 \\
\hline & 11 & $38^{\circ} 00^{\prime} 19^{\prime \prime}$ & $1.28^{\prime} 57^{\prime \prime}$ & 1024 & 20 & 16,2 \\
\hline & 17 & $37^{\circ} 59^{\prime}+8^{\prime \prime}$ & $1^{\circ} 26^{\prime} 31^{\prime \prime}$ & 1045 & 14 & 17,0 \\
\hline & 18 & $37^{\circ} 57^{\prime} 50^{\prime \prime}$ & $1^{\circ} 26^{\prime} 55^{\prime \prime}$ & 1108 & 250 & 18,2 \\
\hline & 19 & $37^{\circ} 55^{\prime} 37^{\prime \prime}$ & $1027^{\prime} 54^{\prime \prime}$ & 1125 & 525 & 18,6 \\
\hline & 20 & $37^{\circ} 53^{\prime} 50^{\prime \prime}$ & $1 \circ 32^{\prime} 42^{\prime \prime}$ & 1220 & 1100 & 19,7 \\
\hline & 21 & $37^{\circ} 54^{\prime} 25^{\prime \prime}$ & $1{ }^{\circ} 31^{\prime} 38^{\prime \prime}$ & 1250 & 780 & 20,2 \\
\hline & 22 & $37^{\circ} 55^{\prime} 36^{\prime \prime}$ & $1^{\circ} 32^{\prime} 04^{\prime \prime}$ & 1305 & 645 & 20,2 \\
\hline & 23 & $37^{\circ} 56^{\prime} 31^{\prime \prime}$ & $1^{\circ} 34^{\prime} 11^{\prime \prime}$ & 1325 & 575 & 19,5 \\
\hline & 24 & $37^{\circ} 56^{\prime} 02^{\prime \prime}$ & $1^{\circ} 37^{\prime} 18^{\prime \prime}$ & 1345 & 420 & 19,0 \\
\hline & 25 & $37^{\circ} 57^{\prime} 57^{\prime \prime}$ & $1^{\circ} 39^{\prime} 08^{\prime \prime}$ & 1410 & 335 & 19,0 \\
\hline & 26 & $37^{\circ} 59^{\prime} 15^{\prime \prime}$ & $1^{\circ} 38^{\prime} 38^{\prime \prime}$ & 1420 & 170 & 19,5 \\
\hline & 27 & $38^{\circ} 00^{\prime} 39^{\prime \prime}$ & $1^{\circ} 39^{\prime} 10^{\prime \prime}$ & 1435 & 40 & 19,5 \\
\hline & 28 & $38^{\circ} 01^{\prime} 32^{\prime \prime}$ & $1.35^{\prime} 54^{\prime \prime}$ & 1457 & 25 & 20,5 \\
\hline & $B$ & $38^{\circ} 01^{\prime} 32^{\prime \prime}$ & $1.34^{\prime} 35^{\prime \prime}$ & 1520 & 160 & 20,1 \\
\hline IV & B & $38^{\circ} 01^{\prime} 32^{\prime \prime}$ & $1^{\circ} 34^{\prime} 35^{\prime \prime}$ & 757 & 160 & 16,0 \\
\hline \multirow{14}{*}{$3-I I I-52$} & 28 & $38^{\circ} 01^{\prime} 32^{\prime \prime}$ & $1435^{\prime} 54^{\prime \prime}$ & 815 & 25 & 16,0 \\
\hline & 27 & $38^{\circ} 00^{\prime} 39^{\prime \prime}$ & $1^{\circ} 39^{\prime} 10^{\prime \prime}$ & 831 & 40 & 16,0 \\
\hline & 29 & $38^{\circ} 00^{\prime} 56^{\prime \prime}$ & $1^{\circ} 40^{\prime} 28^{\prime \prime}$ & 847 & 43 & 16,1 \\
\hline & 30 & $37^{\circ} 59^{\prime} 39^{\prime \prime}$ & $1^{\circ} 41^{\prime} 21^{\prime \prime}$ & 921 & 615 & 15,7 \\
\hline & 31 & $37^{\circ} 59^{\prime} 55^{\prime \prime}$ & $1^{\circ} 43^{\prime} 47^{\prime \prime}$ & 1006 & 95 & 15,0 \\
\hline & 32 & $37^{\circ} 57^{\prime} 09^{\prime \prime}$ & $1^{\circ}+3^{\prime} 25^{\prime \prime}$ & 1030 & 485 & 16,7 \\
\hline & 33 & $37^{\circ} 54^{\prime} 56^{\prime \prime}$ & $1^{\circ} 44^{\prime} 20^{\prime \prime}$ & 1055 & 955 & 18,0 \\
\hline & 34 & $38^{\circ} 00^{\prime} 50^{\prime \prime}$ & $1^{\circ}+7^{\prime} 01^{\prime \prime}$ & 1230 & 50 & 21,5 \\
\hline & 35 & $37^{\circ} 58^{\prime} 25^{\prime \prime}$ & $1^{\circ} 47^{\prime} 10^{\prime \prime}$ & 1300 & 520 & 22,0 \\
\hline & 36 & $38^{\circ} 00^{\prime} 22^{\prime \prime}$ & $1^{\circ} 52^{\prime} 19^{\prime \prime}$ & 1350 & 50 & 22,0 \\
\hline & 37 & $37^{\circ} 59^{\prime} 18^{\prime \prime}$ & $1^{\circ} 53^{\prime} 33^{\prime \prime}$ & 1440 & 250 & 22,5 \\
\hline & 38 & $37^{\circ} 57^{\prime} 37^{\prime \prime}$ & $1^{\circ} 53^{\prime} 02^{\prime \prime}$ & 1655 & 590 & 21,5 \\
\hline & 39 & $37^{\circ} 56^{\prime} 10^{\prime \prime}$ & $1 \circ 54^{\prime} 17^{\prime \prime}$ & 1725 & 826 & 19,5 \\
\hline & $B$ & $38^{\circ} 01^{\prime} 32^{\prime \prime}$ & $1^{\circ} 34^{\prime} 35^{\prime \prime}$ & 1945 & 160 & 16,5 \\
\hline
\end{tabular}




\begin{tabular}{|c|c|c|c|c|c|c|}
\hline Circuito & St. & $\stackrel{N}{N}$ & $\begin{array}{c}\lambda \mathrm{E} \\
\text { (M. Mario) }\end{array}$ & $\mathrm{h} \mathrm{m}$ & $\begin{array}{l}\text { altitudine } \\
\text { in }\end{array}$ & $\begin{array}{l}\text { Temp. } \\
\text { in C. }\end{array}$ \\
\hline $\begin{array}{c}V \\
4-I I I-52\end{array}$ & $\begin{array}{r}\text { B } \\
10 \\
17 \\
19 \\
40 \\
41 \\
42 \\
43 \\
44 \\
45 \\
46 \\
47 \\
48 \\
49 \\
50 \\
51 \\
52 \\
53 \\
54 \\
55 \\
56 \\
57 \\
58 \\
59 \\
60 \\
61 \\
62 \\
38 \\
B\end{array}$ & $\begin{array}{l}38^{\circ} 01^{\prime} 32^{\prime \prime} \\
38^{\circ} 01^{\prime} 28^{\prime \prime} \\
37^{\circ} 59^{\prime} 48^{\prime \prime} \\
37^{\circ} 55^{\prime} 37^{\prime \prime} \\
37^{\circ} 54^{\prime} 24^{\prime \prime} \\
37^{\circ} 52^{\prime} 36^{\prime \prime} \\
37^{\circ} 51^{\prime} 18^{\prime \prime} \\
37^{\circ} 50^{\prime} 19^{\prime \prime} \\
37^{\circ} 48^{\prime} 23^{\prime \prime} \\
37^{\circ} 46^{\prime} 17^{\prime \prime} \\
37^{\circ} 45^{\prime} 25^{\prime \prime} \\
37^{\circ} 47^{\prime} 04^{\prime \prime} \\
37^{\circ} 48^{\prime} 57^{\prime \prime} \\
37^{\circ} 50^{\prime} 20^{\prime \prime} \\
37^{\circ} 48^{\prime} 07^{\prime \prime} \\
37^{\circ} 47^{\prime} 22^{\prime \prime} \\
37^{\circ} 47^{\prime} 31^{\prime \prime} \\
37^{\circ} 48^{\prime} 51^{\prime \prime} \\
37^{\circ} 48^{\prime} 23^{\prime \prime} \\
37^{\circ} 47^{\prime} 53^{\prime \prime} \\
37^{\circ} 47^{\prime} 13^{\prime \prime} \\
37^{\circ} 45^{\prime} 20^{\prime \prime} \\
37^{\circ} 46^{\prime} 08^{\prime \prime} \\
37^{\circ} 48^{\prime} 09^{\prime \prime} \\
37^{\circ} 50^{\prime} 23^{\prime \prime} \\
37^{\circ} 52^{\prime} 49^{\prime \prime} \\
37^{\circ} 55^{\prime} 25^{\prime \prime} \\
37^{\circ} 57^{\prime} 37^{\prime \prime} \\
38^{\circ} 01^{\prime} 32^{\prime \prime} \\
\end{array}$ & $\begin{array}{l}1^{\circ} 34^{\prime} 35^{\prime \prime} \\
1^{\circ} 30^{\prime} 44^{\prime \prime} \\
1^{\circ} 26^{\prime} 31^{\prime \prime} \\
1^{\circ} 27^{\prime} 54^{\prime \prime} \\
1^{\circ} 28^{\prime} 03^{\prime \prime} \\
1^{\circ} 27^{\prime} 35^{\prime \prime} \\
1^{\circ} 27^{\prime} 52^{\prime \prime} \\
1^{\circ} 26^{\prime} 28^{\prime \prime} \\
1^{\circ} 26^{\prime} 49^{\prime \prime} \\
1^{\circ} 28^{\prime} 50^{\prime \prime} \\
1^{\circ} 32^{\prime} 12^{\prime \prime} \\
1^{\circ} 34^{\prime} 05^{\prime \prime} \\
1^{\circ} 33^{\prime} 011^{\prime \prime} \\
1^{\circ} 30^{\prime} 27^{\prime \prime} \\
1^{\circ} 35^{\prime} 09^{\prime \prime} \\
1^{\circ} 37^{\prime} 17^{\prime \prime} \\
1^{\circ} 39^{\prime} 40^{\prime \prime} \\
1^{\circ} 41^{\prime} 20^{\prime \prime} \\
1^{\circ} 43^{\prime} 57^{\prime \prime} \\
1^{\circ} 48^{\prime} 02^{\prime \prime} \\
1^{\circ} 52^{\prime} 20^{\prime \prime} \\
1^{\circ} 54^{\prime} 36^{\prime \prime} \\
1^{\circ} 57^{\prime} 37^{\prime \prime} \\
1^{\circ} 58^{\prime} 15^{\prime \prime} \\
1^{\circ} 56^{\prime} 49^{\prime \prime} \\
1^{\circ} 55^{\prime} 42^{\prime \prime} \\
1^{\circ} 54^{\prime} 40^{\prime \prime} \\
1^{\circ} 53^{\prime} 02^{\prime \prime} \\
1^{\circ} 35^{\prime}\end{array}$ & $\begin{array}{rl}7 & 46 \\
8 & 10 \\
8 & 30 \\
9 & 00 \\
9 & 22 \\
9 & 41 \\
10 & 00 \\
10 & 26 \\
10 & 53 \\
11 & 11 \\
11 & 28 \\
11 & 44 \\
12 & 04 \\
12 & 24 \\
13 & 05 \\
13 & 29 \\
15 & 17 \\
15 & 31 \\
15 & 51 \\
16 & 11 \\
16 & 26 \\
16 & 46 \\
17 & 34 \\
17 & 51 \\
18 & 10 \\
18 & 27 \\
18 & 53 \\
19 & 24 \\
20 & 45\end{array}$ & $\begin{array}{r}160 \\
45 \\
14 \\
525 \\
560 \\
525 \\
370 \\
445 \\
700 \\
810 \\
825 \\
850 \\
770 \\
550 \\
935 \\
840 \\
1020 \\
975 \\
870 \\
765 \\
674 \\
620 \\
825 \\
924 \\
1120 \\
1075 \\
945 \\
590 \\
160\end{array}$ & $\begin{array}{l}15,7 \\
14,7 \\
15,0 \\
16,0 \\
17,0 \\
18,0 \\
19,02 \\
20,0 \\
21,2 \\
20,0 \\
20,5 \\
20,0 \\
21,0 \\
21,0 \\
21,8 \\
22,0 \\
25,0 \\
24,0 \\
22,0 \\
21,5 \\
20,5 \\
19,1 \\
19,5 \\
18,0 \\
16,7 \\
15,0 \\
13,2 \\
12,0 \\
12,0\end{array}$ \\
\hline $\begin{array}{c}\text { VI } \\
5-I I I-52\end{array}$ & $\begin{array}{l}\text { B } \\
63 \\
64 \\
65 \\
66 \\
67 \\
68 \\
69 \\
70 \\
71 \\
72 \\
73 \\
74 \\
75 \\
76 \\
77 \\
78 \\
79 \\
80 \\
81 \\
82\end{array}$ & $\begin{array}{l}38^{\circ} 01^{\prime} 32^{\prime \prime} \\
38^{\prime \prime} 02^{\prime} 01^{\prime \prime} \\
38^{\circ} 01^{\prime} 01^{\prime \prime} \\
37^{\circ} 56^{\prime} 15^{\prime \prime} \\
37^{\circ} 54^{\prime} 54^{\prime \prime} \\
37^{\circ} 53^{\prime} 21^{\prime \prime} \\
37^{\circ} 51^{\prime} 58^{\prime \prime} \\
37^{\circ} 49^{\prime} 32^{\prime \prime} \\
37^{\circ} 46^{\prime} 35^{\prime \prime} \\
37^{\circ} 44^{\prime} 02^{\prime \prime} \\
37^{\circ} 42^{\prime} 08^{\prime \prime} \\
37^{\circ} 40^{\prime} 58^{\prime \prime} \\
37^{\circ} 40^{\prime} 43^{\prime \prime} \\
37^{\circ} 39^{\prime} 36^{\prime \prime} \\
37^{\circ} 37^{\prime} 32^{\prime \prime} \\
37^{\circ} 35^{\prime} 15^{\prime \prime} \\
37^{\circ} 34^{\prime} 58^{\prime \prime} \\
37^{\circ} 32^{\prime} 42^{\prime \prime} \\
37^{\circ} 30^{\prime} 03^{\prime \prime} \\
37^{\circ} 29^{\prime} 06^{\prime \prime} \\
37^{\circ} 26^{\prime} 19^{\prime \prime}\end{array}$ & $\begin{array}{l}1^{\circ} 34^{\prime} 35^{\prime \prime} \\
1^{\circ} 33^{\prime} 29^{\prime \prime} \\
1^{\circ} 38^{\prime} 11^{\prime \prime} \\
1^{\circ} 38^{\prime} 34^{\prime \prime} \\
1^{\circ} 39^{\prime} 25^{\prime \prime} \\
1^{\circ} 41^{\prime} 03^{\prime \prime} \\
1^{\circ} 42^{\prime} 10^{\prime \prime} \\
1^{\circ} 42^{\prime} 08^{\prime \prime} \\
1^{\circ} 38^{\prime} 44^{\prime \prime} \\
1^{\circ} 38^{\prime} 46^{\prime \prime} \\
1^{\circ} 39^{\prime} 50^{\prime \prime} \\
1^{\circ} 38^{\prime} 30^{\prime \prime} \\
1^{\circ} 35^{\prime} 00^{\prime \prime} \\
1^{\circ} 33^{\prime} 55^{\prime \prime} \\
1^{\circ} 32^{\prime} 59^{\prime \prime} \\
1^{\circ} 34^{\prime} 05^{\prime \prime} \\
1^{\circ} 36^{\prime} 48^{\prime \prime} \\
1^{\circ} 36^{\prime} 27^{\prime \prime} \\
1^{\circ} 36^{\prime} 15^{\prime \prime} \\
1^{\circ} 34^{\prime} 33^{\prime \prime} \\
1^{\circ} 35^{\prime} 49^{\prime \prime}\end{array}$ & $\begin{array}{rr}8 & 42 \\
9 & 07 \\
9 & 31 \\
10 & 07 \\
10 & 23 \\
10 & 50 \\
11 & 07 \\
11 & 27 \\
12 & 02 \\
12 & 17 \\
12 & 34 \\
14 & 37 \\
14 & 51 \\
15 & 21 \\
15 & 40 \\
15 & 57 \\
16 & 14 \\
16 & 33 \\
16 & 55 \\
17 & 15 \\
18 & 05\end{array}$ & $\begin{array}{r}160 \\
1 \\
100 \\
359 \\
350 \\
700 \\
970 \\
1100 \\
915 \\
710 \\
760 \\
675 \\
550 \\
830 \\
775 \\
655 \\
685 \\
480 \\
650 \\
650 \\
385\end{array}$ & $\begin{array}{l}16,0 \\
17,0 \\
17,5 \\
18,0 \\
18,7 \\
19,0 \\
19,5 \\
19,5 \\
19,5 \\
19,0 \\
18,7 \\
21,0 \\
19,7 \\
20,0 \\
19,5 \\
19,0 \\
19,0 \\
18,7 \\
19,5 \\
19,0 \\
18,0\end{array}$ \\
\hline
\end{tabular}




\begin{tabular}{|c|c|c|c|c|c|c|}
\hline Circuito & St. & $\varphi \mathrm{N}$ & $\begin{array}{c}\lambda E \\
\text { (M. Mario) }\end{array}$ & h $m$ & $\begin{array}{l}\text { altitudine } \\
\text { In }\end{array}$ & $\begin{array}{l}\text { Temp. } \\
\text { in Co }\end{array}$ \\
\hline \multirow[t]{19}{*}{$6-I I I-52$} & 82 & $37^{\circ} 26^{\prime} 19^{\prime \prime}$ & $1035^{\prime}+9^{\prime \prime}$ & 846 & 385 & 18,5 \\
\hline & 78 & $37^{\circ} 3 t^{\prime} 58^{\prime \prime}$ & $1^{\circ} 36^{\prime}+8^{\prime \prime}$ & 955 & 685 & 17,7 \\
\hline & 75 & $37^{\circ} 39^{\prime} 36^{\prime \prime}$ & $1^{\circ} 33^{\prime} 55^{\prime \prime}$ & 1028 & 830 & 18.2 \\
\hline & 83 & $37^{\circ} 39^{\prime} 38^{\prime \prime}$ & $1029^{\prime} 36^{\prime \prime}$ & $10+7$ & 450 & 18,2 \\
\hline & 84 & $37^{\circ} 39^{\prime} 38^{\prime \prime}$ & $1026^{\prime}+4^{\prime \prime}$ & 1106 & 373 & 19,0 \\
\hline & 85 & $37^{\circ}+0^{\prime} 54^{\prime \prime}$ & $1^{\circ} 23^{\prime} 06^{\prime \prime}$ & 1123 & 450 & 19,7 \\
\hline & 86 & $37^{\circ}+1^{\prime} 50^{\prime \prime}$ & $1^{\circ 2} 21^{\prime} 19^{\prime \prime}$ & $11+0$ & 600 & 20.0 \\
\hline & 87 & $37^{\circ}+3^{\prime} 20^{\prime \prime}$ & $10^{\circ} 20^{\prime} 15^{\prime \prime}$ & 1155 & $6+0$ & 20,2 \\
\hline & 88 & $37^{\circ} 44^{\prime}+1^{\prime \prime}$ & $1^{\circ} 22^{\prime} 04^{\prime \prime}$ & 1218 & 775 & 20,2 \\
\hline & 89 & $37^{\circ}+5^{\prime} 28^{\prime \prime}$ & $1^{\circ} 26^{\prime} 17^{\prime \prime}$ & $12+9$ & 825 & 20,7 \\
\hline & 90 & $37^{\circ}+7^{\prime}+1^{\prime \prime}$ & $1 \circ 26^{\prime} 24^{\prime \prime}$ & 1306 & 750 & 20,2 \\
\hline & 91 & $37^{\circ} 50^{\prime} 00^{\prime \prime}$ & $1^{\circ} 24^{\prime} 51^{\prime \prime}$ & 1504 & 375 & 21,5 \\
\hline & 92 & $37^{\circ} 51^{\prime}+8^{\prime \prime}$ & $1 \circ 23^{\prime} 50^{\prime \prime}$ & 1526 & 350 & 21,5 \\
\hline & 93 & $37^{\circ} 53^{\prime} 54^{\prime \prime}$ & $1^{\circ} 21^{\prime}+1^{\prime \prime}$ & 1543 & 260 & 20,0 \\
\hline & 94 & $37^{\circ} 55^{\prime} 33^{\prime \prime}$ & $1^{\circ} 20^{\prime} 19^{\prime \prime}$ & 1616 & 115 & 19,5 \\
\hline & 95 & $37^{\circ} 57^{\prime} 48^{\prime \prime}$ & $1^{\circ} 20^{\prime} 38^{\prime \prime}$ & 1630 & 30 & 19,5 \\
\hline & 96 & $37^{\circ} 58^{\prime}+9^{\prime \prime}$ & $1023^{\prime}+5^{\prime \prime}$ & $16+6$ & 10 & 19,2 \\
\hline & 97 & $38^{\circ} 00^{\prime} 57^{\prime \prime}$ & $10^{\circ} 28^{\prime} 24^{\prime \prime}$ & 1718 & + & 19,7 \\
\hline & B & $38^{\circ} 01^{\prime} 32^{\prime \prime}$ & $1.34^{\prime} 35^{\prime \prime}$ & $17+5$ & 160 & 19,0 \\
\hline \multirow[t]{2}{*}{ VII } & B & $38^{\circ} 01^{\prime} 32^{\prime \prime}$ & $1 \circ 3+35^{\prime \prime}$ & 911 & 160 & 16,5 \\
\hline & 93 & $37^{\circ} 53^{\prime} 54^{\prime \prime}$ & $1^{\circ} 21^{\prime}+1^{\prime \prime}$ & 1006 & 260 & 17,2 \\
\hline \multirow[t]{17}{*}{$7-I I I-52$} & 98 & $37^{\circ} 51^{\prime} 57^{\prime \prime}$ & $1{ }^{\circ} 20^{\prime} 31^{\prime \prime}$ & 1025 & 430 & 17,6 \\
\hline & 99 & $37^{\circ} 49^{\prime} 51^{\prime \prime}$ & $1^{\circ} 18^{\prime} 58^{\prime \prime}$ & $10+4$ & 620 & 18,0 \\
\hline & 100 & $37^{\circ} 48^{\prime} 11^{\prime \prime}$ & $1^{\circ} 17^{\prime} 35^{\prime \prime}$ & 1102 & 627 & 18,0 \\
\hline & 101 & $37^{\circ} 44^{\prime} 53^{\prime \prime}$ & $1^{\circ} 17^{\prime} 03^{\prime \prime}$ & $11+8$ & 550 & 18,7 \\
\hline & 102 & $37^{\circ}+6^{\prime} 22^{\prime \prime}$ & $1^{\circ} 15^{\prime} 00^{\prime \prime}$ & 1202 & 535 & 19,0 \\
\hline & 103 & $37^{\circ}+7^{\prime}+0^{\prime \prime}$ & $1^{\circ} 12^{\prime} 01^{\prime \prime}$ & 1221 & 390 & 19,0 \\
\hline & 104 & $37^{\circ} 48^{\prime} 54^{\prime \prime}$ & $1^{\circ} 11^{\prime} 08^{\prime \prime}$ & $12+2$ & 580 & 20,5 \\
\hline & 105 & $37^{\prime \prime}+7^{\prime} 35^{\prime \prime}$ & $1^{\circ} 09^{\prime} 00^{\prime \prime}$ & 1304 & 685 & 21,1 \\
\hline & 106 & $37^{\circ}+9^{\prime} 16^{\prime \prime}$ & $1^{\circ} 06^{\prime}+2^{\prime \prime}$ & 1408 & 575 & 20,0 \\
\hline & 107 & $37^{\circ} 51^{\prime} 09^{\prime \prime}$ & $1^{\circ} 03^{\prime} 58^{\prime \prime}$ & $1+25$ & 290 & 20,7 \\
\hline & 108 & $37^{\circ} 53^{\prime} 54^{\prime \prime}$ & $1^{\circ} 01^{\prime} 48^{\prime \prime}$ & $1+42$ & 530 & 20,5 \\
\hline & 109 & $37^{\circ} 55^{\prime}+6^{\prime \prime}$ & $1^{\circ} 01^{\prime} 57^{\prime \prime}$ & $1+58$ & 410 & 20,5 \\
\hline & 110 & $37^{\circ} 54^{\prime} 57^{\prime \prime}$ & $1^{\circ} 05^{\prime} 04^{\prime \prime}$ & 1519 & 575 & 20,5 \\
\hline & 111 & $37^{\circ} 5 t^{\prime} 45^{\prime \prime}$ & $1^{\circ} 06^{\prime} 32^{\prime \prime}$ & 1535 & 402 & 19,7 \\
\hline & 112 & $37^{\circ} 56^{\prime} 17^{\prime \prime}$ & $1^{\circ} 09^{\prime} 34^{\prime \prime}$ & 1600 & 450 & 19,0 \\
\hline & 113 & $37^{\circ} 59^{\prime} 31^{\prime \prime}$ & $1 \circ 10^{\prime} 57^{\prime \prime}$ & 1615 & 175 & 18,5 \\
\hline & B & $38^{\circ} 01^{\prime} 32^{\prime \prime}$ & $1 \circ 34^{\prime} 35^{\prime \prime}$ & 2001 & 160 & 18,0 \\
\hline \multirow{3}{*}{$\begin{array}{c}\text { VIII } \\
8-I I I-52\end{array}$} & B & $38^{\circ} 01^{\prime} 32^{\prime \prime}$ & $1034^{\prime} 35^{\prime \prime}$ & $\begin{array}{lrr}\text { (H) } & 9 & 19 \\
\text { (Z) } & 10 & 33\end{array}$ & 160 & 17,0 \\
\hline & 114 & $38^{\circ} 00^{\prime} 22^{\prime \prime}$ & $1 \circ 53^{\prime} 29^{\prime \prime}$ & (C) $\begin{array}{rl}10 & 33 \\
11 & +2\end{array}$ & 30 & 21,7 \\
\hline & B & $38^{\circ} 01^{\prime} 32^{\prime \prime}$ & $1 \circ 34^{\prime} 35^{\prime \prime}$ & 1537 & 160 & 21,7 \\
\hline \multirow[t]{2}{*}{ IX } & $B$ & $38^{\circ} 01^{\prime} 32^{\prime \prime}$ & $1 \circ 34^{\prime} 35^{\prime \prime}$ & $7+2$ & 160 & 15,0 \\
\hline & 115 & $37^{\circ} 56^{\prime} 36^{\prime \prime}$ & $1^{\circ} 19^{\prime} 17^{\prime \prime}$ & 830 & 110 & 13,5 \\
\hline \multirow[t]{10}{*}{$10-11 \mathrm{I}-52$} & 116 & $37^{\circ} 5 t^{\prime} 36^{\prime \prime}$ & $1^{\circ} 18^{\prime} 15^{\prime \prime}$ & 854 & 270 & 13,5 \\
\hline & 117 & $37^{\circ} 5 t^{\prime} 18^{\prime \prime}$ & $1^{\circ} 15^{\prime} 12^{\prime \prime}$ & 917 & 465 & 13,0 \\
\hline & 118 & $37^{\circ} 54^{\prime} 10^{\prime \prime}$ & $1^{\circ} 13^{\prime} 10^{\prime \prime}$ & $9+2$ & 550 & 13,2 \\
\hline & 119 & $37^{\circ} 52^{\prime} 33^{\prime \prime}$ & $1^{\circ} 11^{\prime} 35^{\prime \prime}$ & 1005 & 585 & 13,2 \\
\hline & 120 & $37^{\circ} 50^{\prime} 28^{\prime \prime}$ & $1^{\circ} 11^{\prime} 03^{\prime \prime}$ & 1026 & 455 & 12,5 \\
\hline & 121 & $37^{\circ} 56^{\prime} 38^{\prime \prime}$ & $1012^{\prime} 48^{\prime \prime}$ & 1106 & 460 & 13,2 \\
\hline & 122 & $37^{\circ} 58^{\prime} 27^{\prime \prime}$ & $1^{\circ} 14^{\prime} 03^{\prime \prime}$ & 1131 & 95 & 12,7 \\
\hline & 123 & $37^{\circ} 58^{\prime} 13^{\prime \prime}$ & $1^{\circ} 16^{\prime} 54^{\prime \prime}$ & 1154 & 70 & 15,2 \\
\hline & 124 & $38^{\circ} 06^{\prime} 13^{\prime \prime}$ & $10 n 4^{\prime} 56^{N}$ & $14+2$ & 12 & 20,2 \\
\hline & $B$ & $38^{\circ} 01^{\prime} 32^{\prime \prime}$ & $1034^{\prime} 35^{\prime \prime}$ & 1610 & 160 & 19,7 \\
\hline
\end{tabular}


Tabella n. 4.

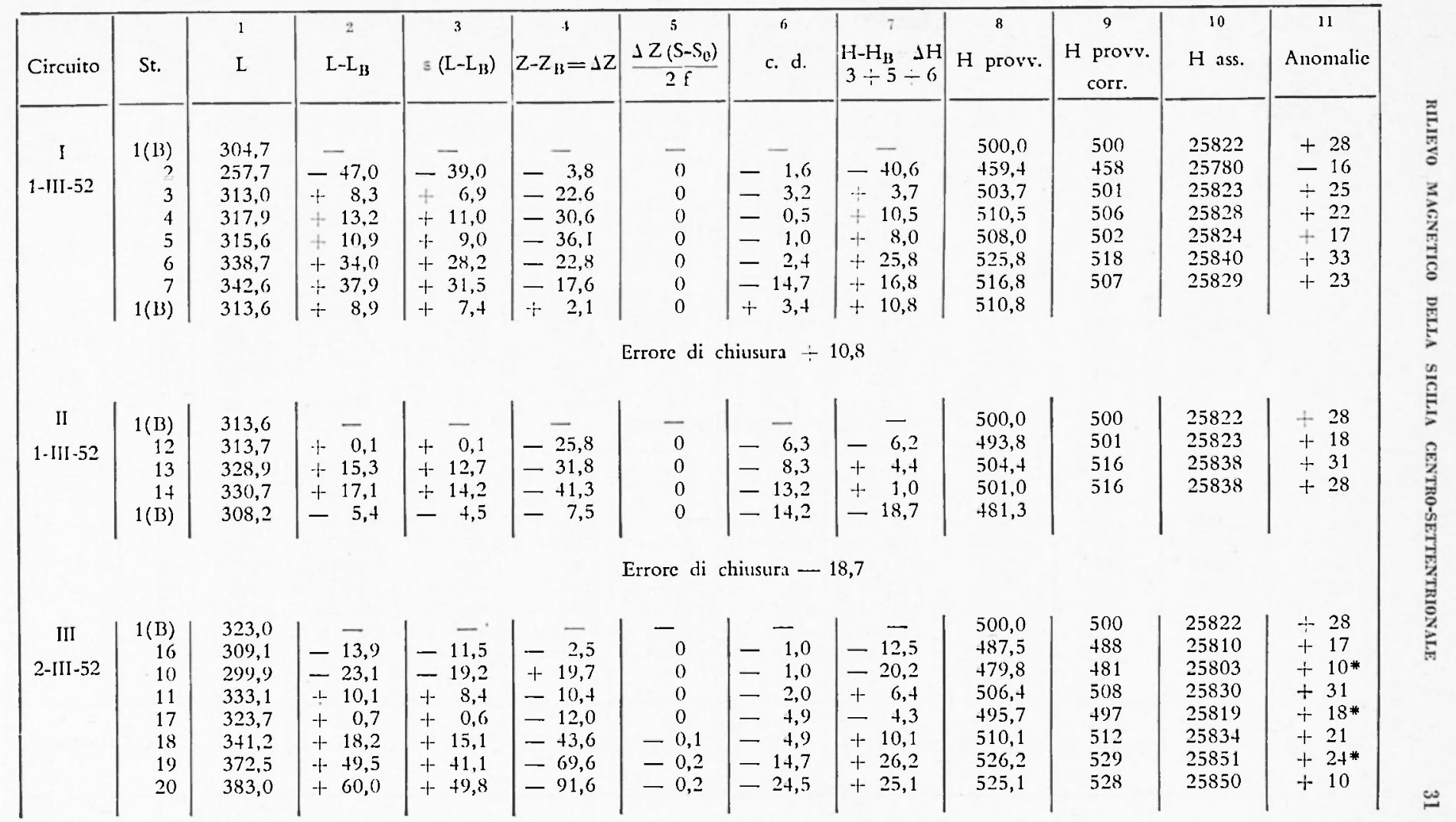




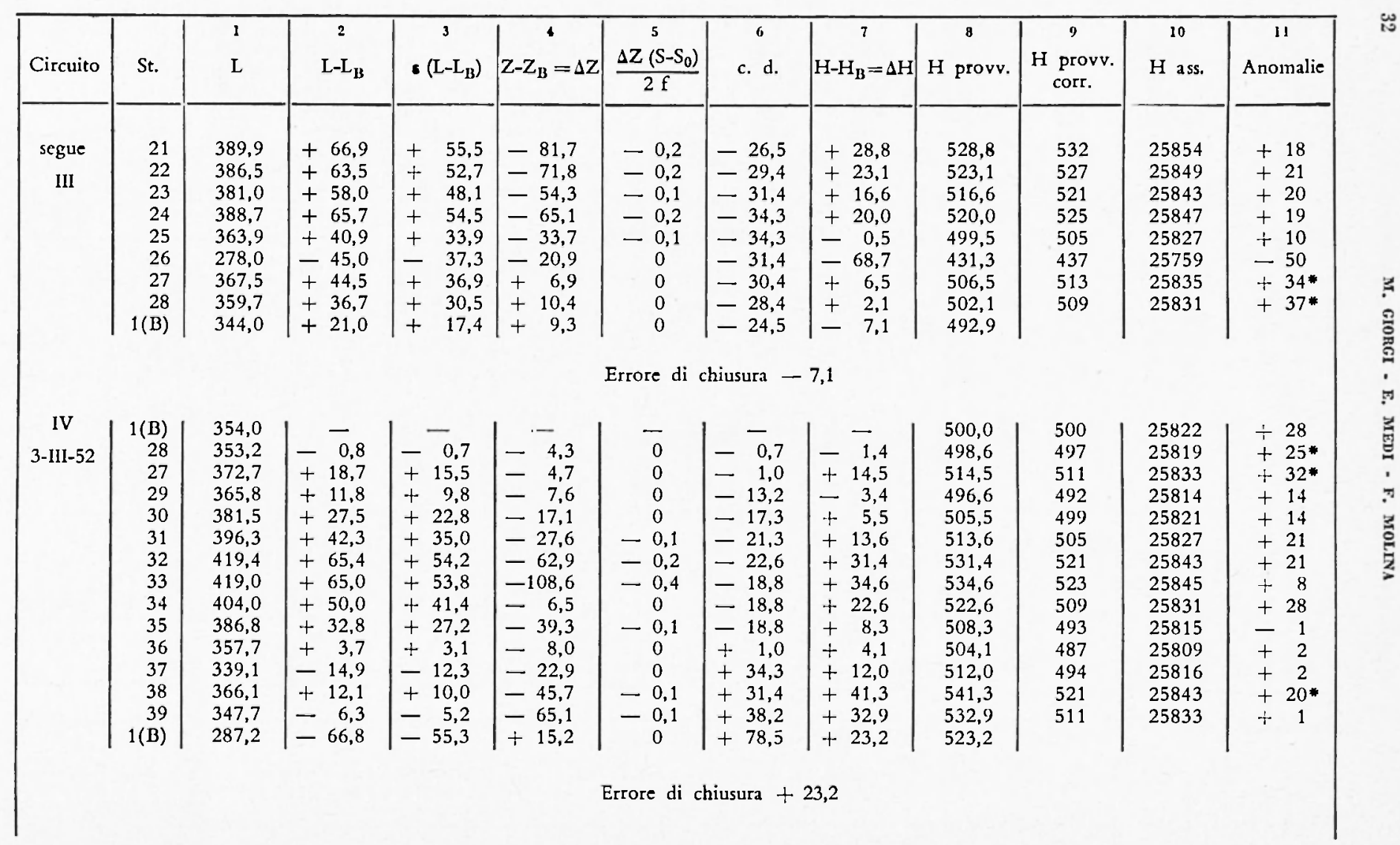




\begin{tabular}{|c|c|c|}
\hline 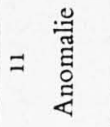 & 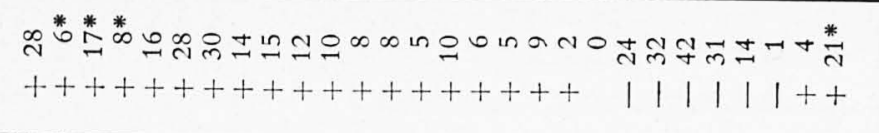 & \\
\hline$\stackrel{\stackrel{\dot{g}}{g}}{\check{I}}$ & 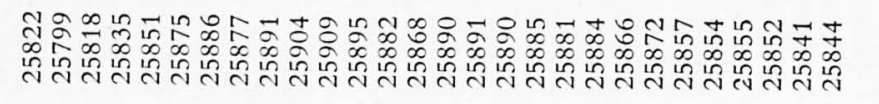 & \\
\hline 荾宽 & 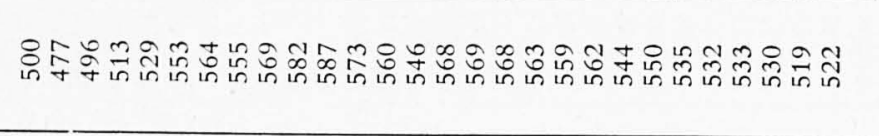 & \\
\hline$\infty \begin{array}{l}\stackrel{3}{0} \\
\text { 岁 } \\
\text { 生 }\end{array}$ & 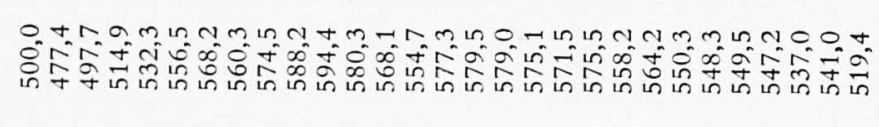 & \\
\hline ‥ & 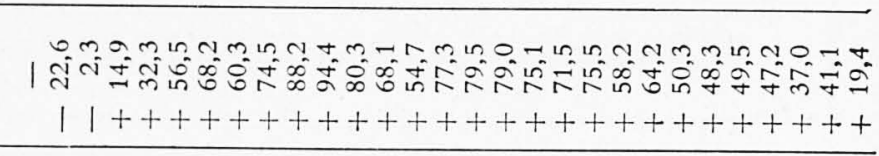 & ळ. \\
\hline $\begin{array}{ll}\dot{\tau} \\
\end{array}$ & 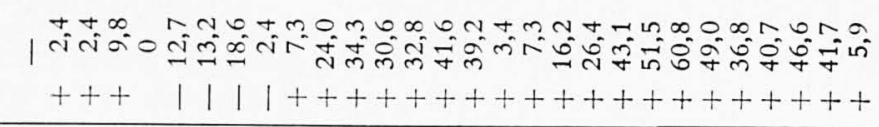 & 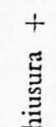 \\
\hline 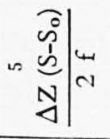 & 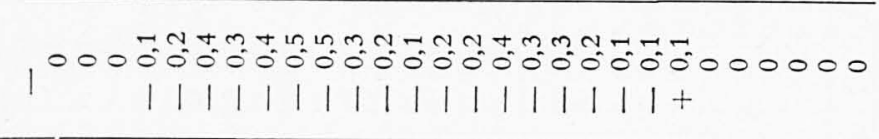 & 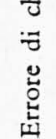 \\
\hline $\begin{array}{c}N \\
11 \\
N \\
N \\
N \\
N\end{array}$ & 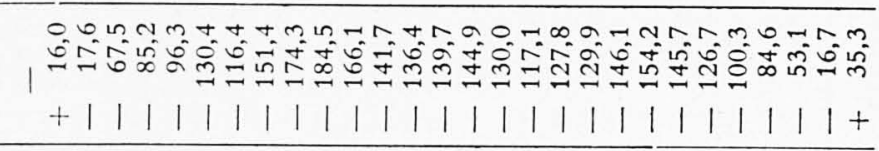 & \\
\hline$\underbrace{\frac{\omega_{1}^{3}}{e}}_{\omega}$ & 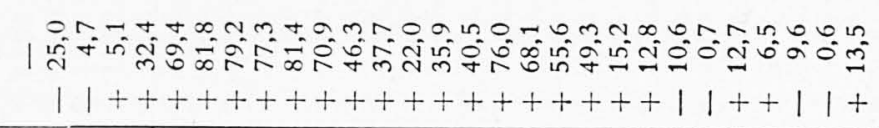 & \\
\hline ब & 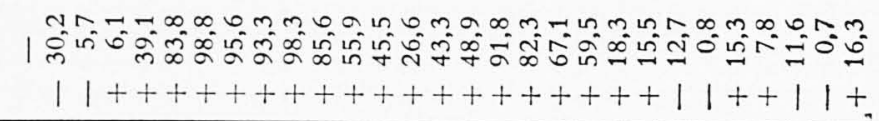 & \\
\hline$-H$ & 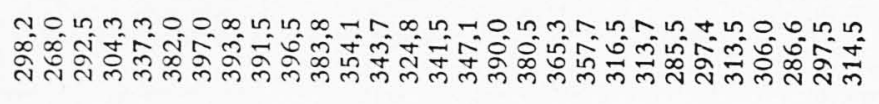 & \\
\hline$\ddot{n}$ & 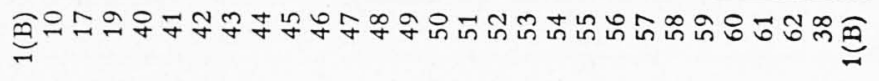 & \\
\hline 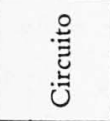 & 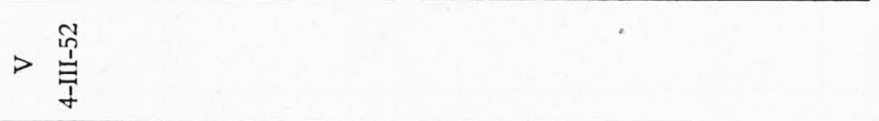 & \\
\hline
\end{tabular}




\begin{tabular}{|c|c|}
\hline 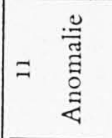 & 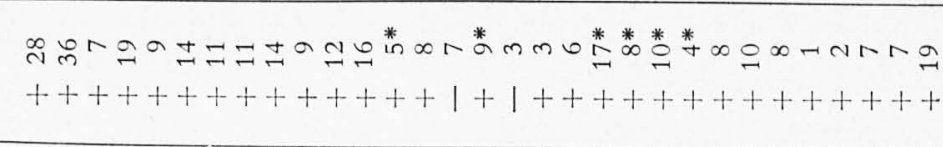 \\
\hline 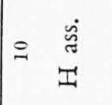 & 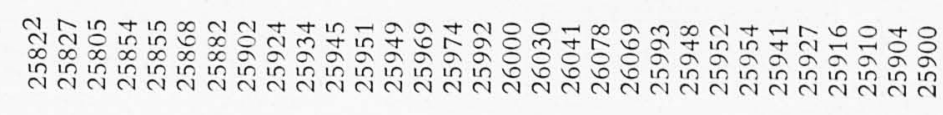 \\
\hline 竞容 & 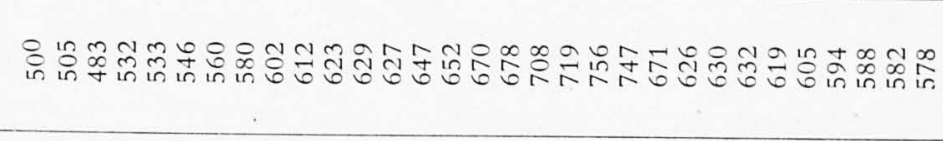 \\
\hline 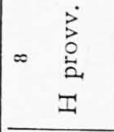 & 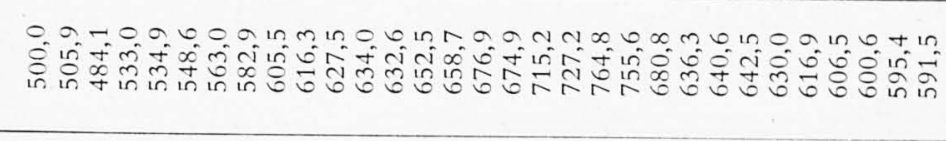 \\
\hline 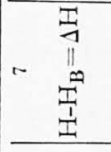 & 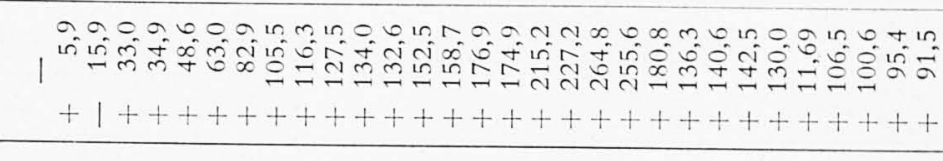 \\
\hline $\begin{array}{l}0 \quad \dot{\tau} \\
\dot{u}\end{array}$ & 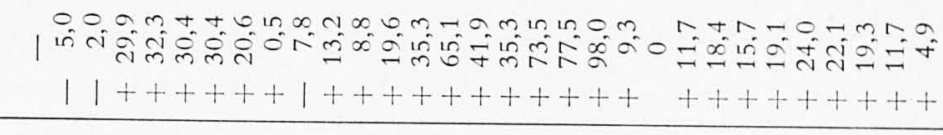 \\
\hline 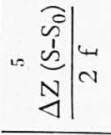 & 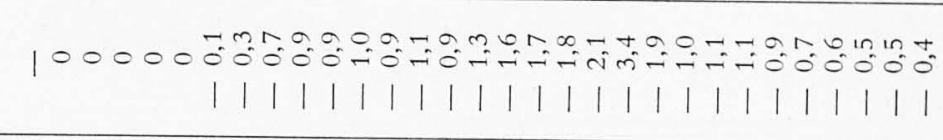 \\
\hline$+\underset{\substack{N \\
N}}{N}$ & 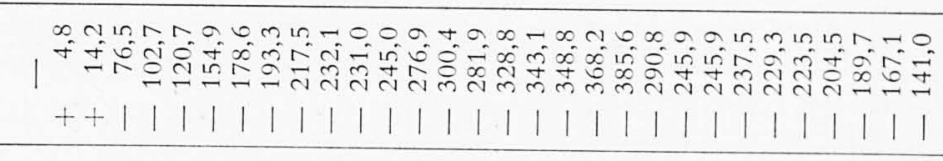 \\
\hline 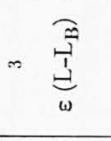 & 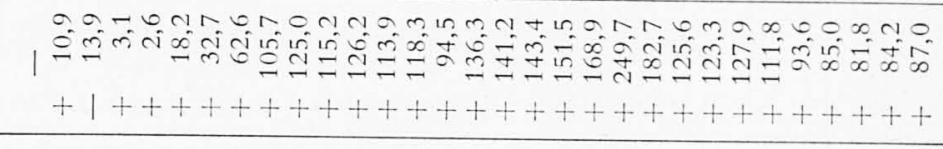 \\
\hline$\Rightarrow \stackrel{\text { जै }}{4}$ & 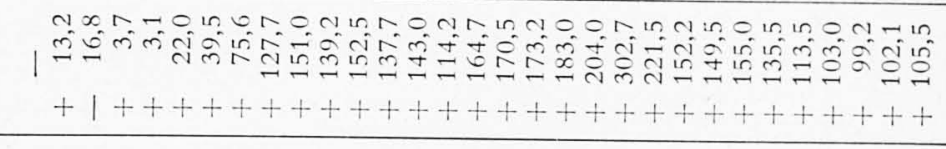 \\
\hline$-\omega$ & 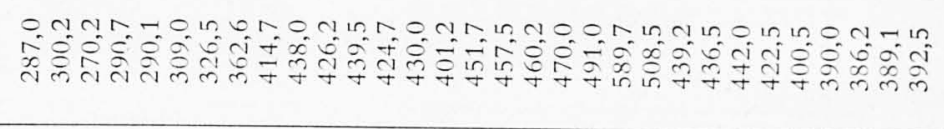 \\
\hline$\dot{\omega}$ & 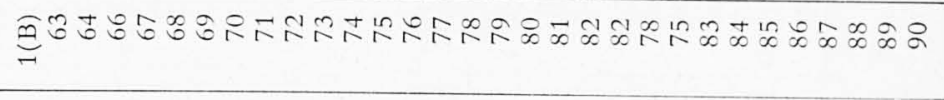 \\
\hline 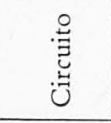 & 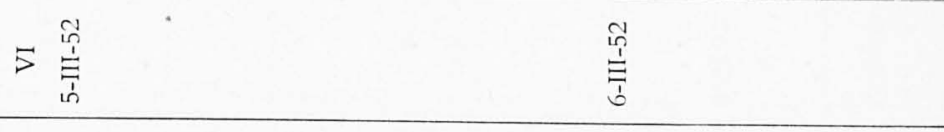 \\
\hline
\end{tabular}




\begin{tabular}{|c|c|c|c|}
\hline$=\underset{\check{\Xi}}{\stackrel{\cong}{\Xi}}$ & 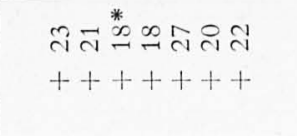 & & 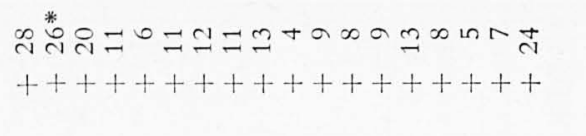 \\
\hline 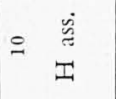 & 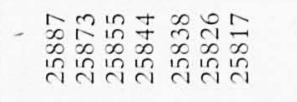 & & 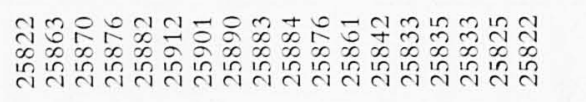 \\
\hline 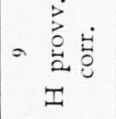 & 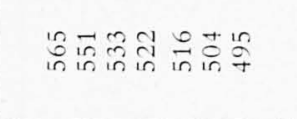 & & 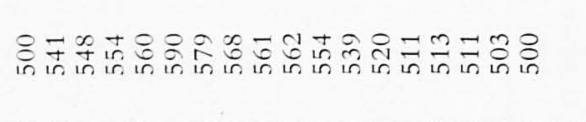 \\
\hline 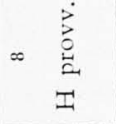 & 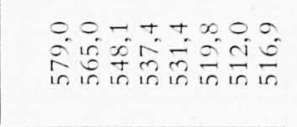 & & 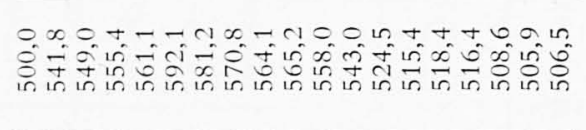 \\
\hline 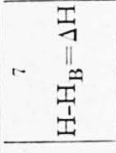 & 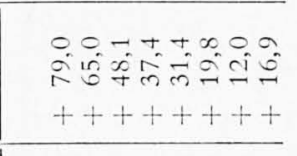 & छे & 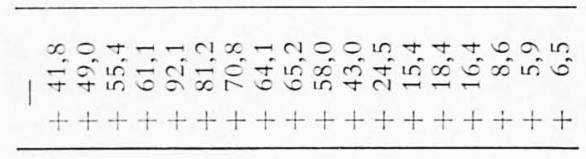 \\
\hline - $\quad \dot{j}$ & 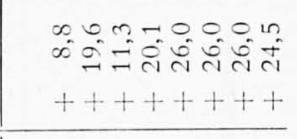 & $\begin{array}{l}+ \\
\ddot{\Xi} \\
\stackrel{\Xi}{\Xi}\end{array}$ & 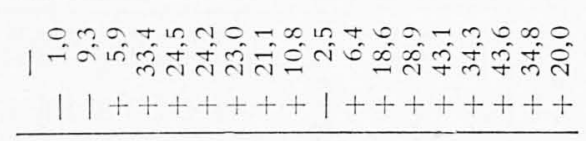 \\
\hline 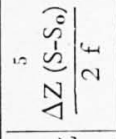 & $\begin{array}{l}3=0000000 \\
0000 \\
|1|\end{array}$ & 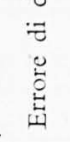 & 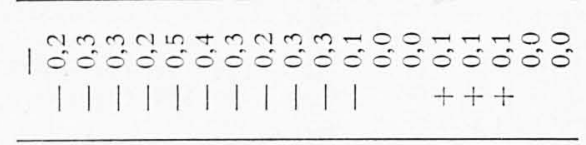 \\
\hline $\begin{array}{r}N \\
11 \\
+N \\
N \\
N \\
N\end{array}$ & 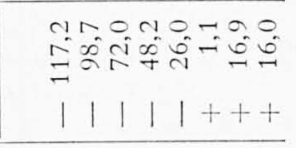 & & 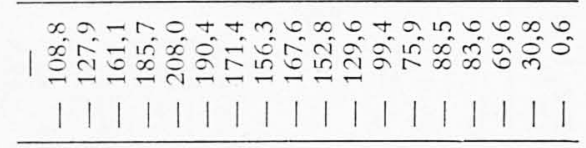 \\
\hline$\therefore \underset{\omega}{\stackrel{0}{*}}$ & 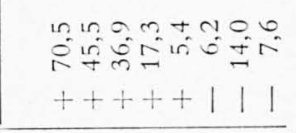 & & 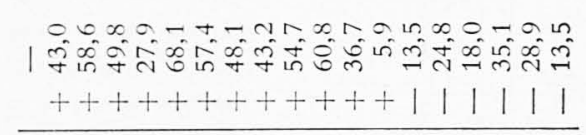 \\
\hline$\therefore \frac{\hat{7}}{4}$ & 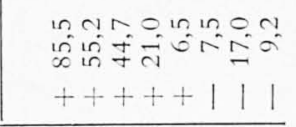 & & 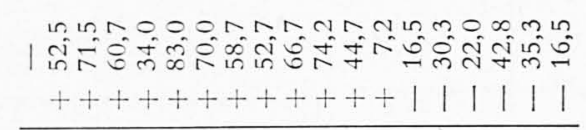 \\
\hline-1 & 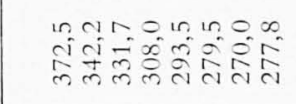 & & 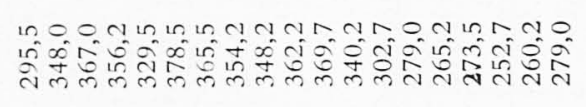 \\
\hline$\dot{n}$ & 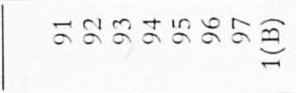 & & 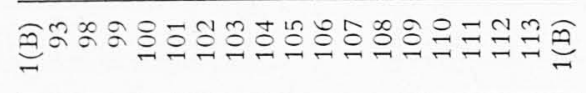 \\
\hline 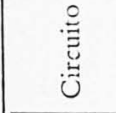 & 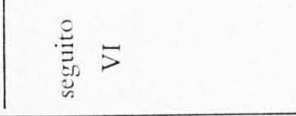 & & 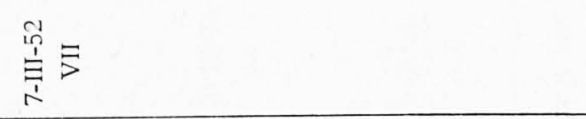 \\
\hline
\end{tabular}




\begin{tabular}{|c|c|c|c|c|}
\hline 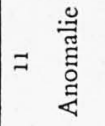 & $\begin{array}{l}\stackrel{\sim}{\sim} \\
++\end{array}$ & \multirow{13}{*}{ 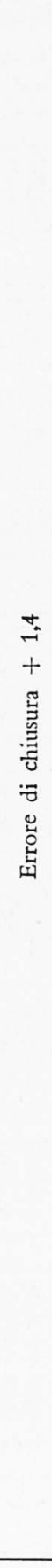 } & 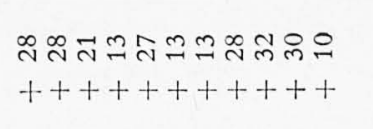 & \multirow{13}{*}{ 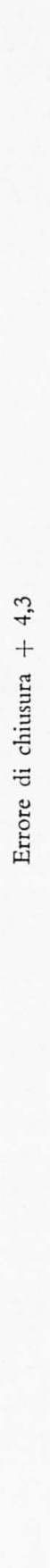 } \\
\hline$=\begin{array}{c}\dot{m} \\
\dot{m} \\
\dot{I}\end{array}$ & $\begin{array}{l}\text { तै } \\
\infty \\
\stackrel{\infty}{\infty} \\
\stackrel{n}{\sim}\end{array}$ & & 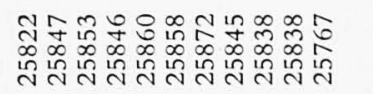 & \\
\hline 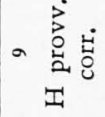 & 응 & & 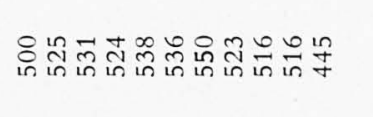 & \\
\hline 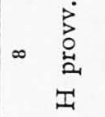 & 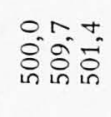 & & 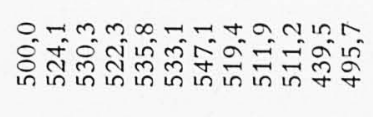 & \\
\hline 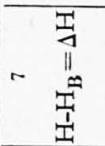 & $\begin{array}{l}\hat{\sigma}= \\
++\end{array}$ & & 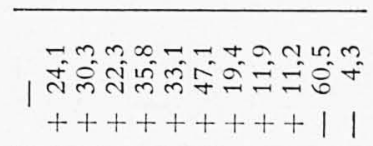 & \\
\hline - $\quad \dot{j}$ & $\begin{array}{l}\text { in } \stackrel{\text { a }}{-i} \\
++\end{array}$ & & 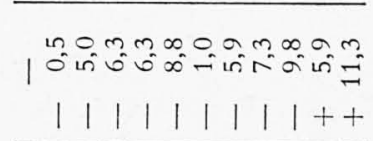 & \\
\hline 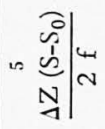 & 100 & & 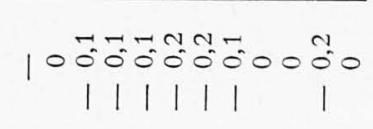 & \\
\hline $\begin{array}{r}N \\
-\quad \|^{m} \\
N \\
N \\
N\end{array}$ & 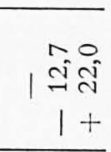 & & 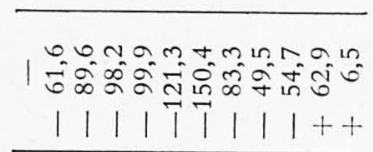 & \\
\hline$m \underbrace{\stackrel{a}{!}}_{\omega}$ & $\begin{array}{l}1_{\substack{x \\
\infty}}^{\infty}+1 \\
\end{array}$ & & 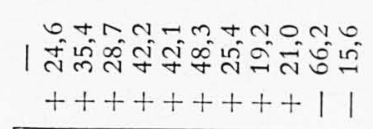 & \\
\hline$\sim \stackrel{\rightarrow}{\leftrightarrow}$ & $\begin{array}{l}0 \infty \\
1 \stackrel{\infty}{0}=- \\
+1\end{array}$ & & 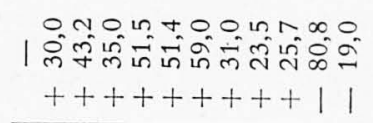 & \\
\hline$-H$ & 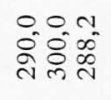 & & 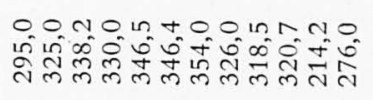 & \\
\hline$\dot{s}$ & 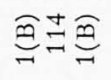 & & 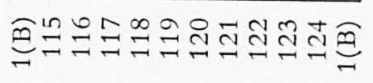 & \\
\hline .: & 志 & & 范 & \\
\hline
\end{tabular}




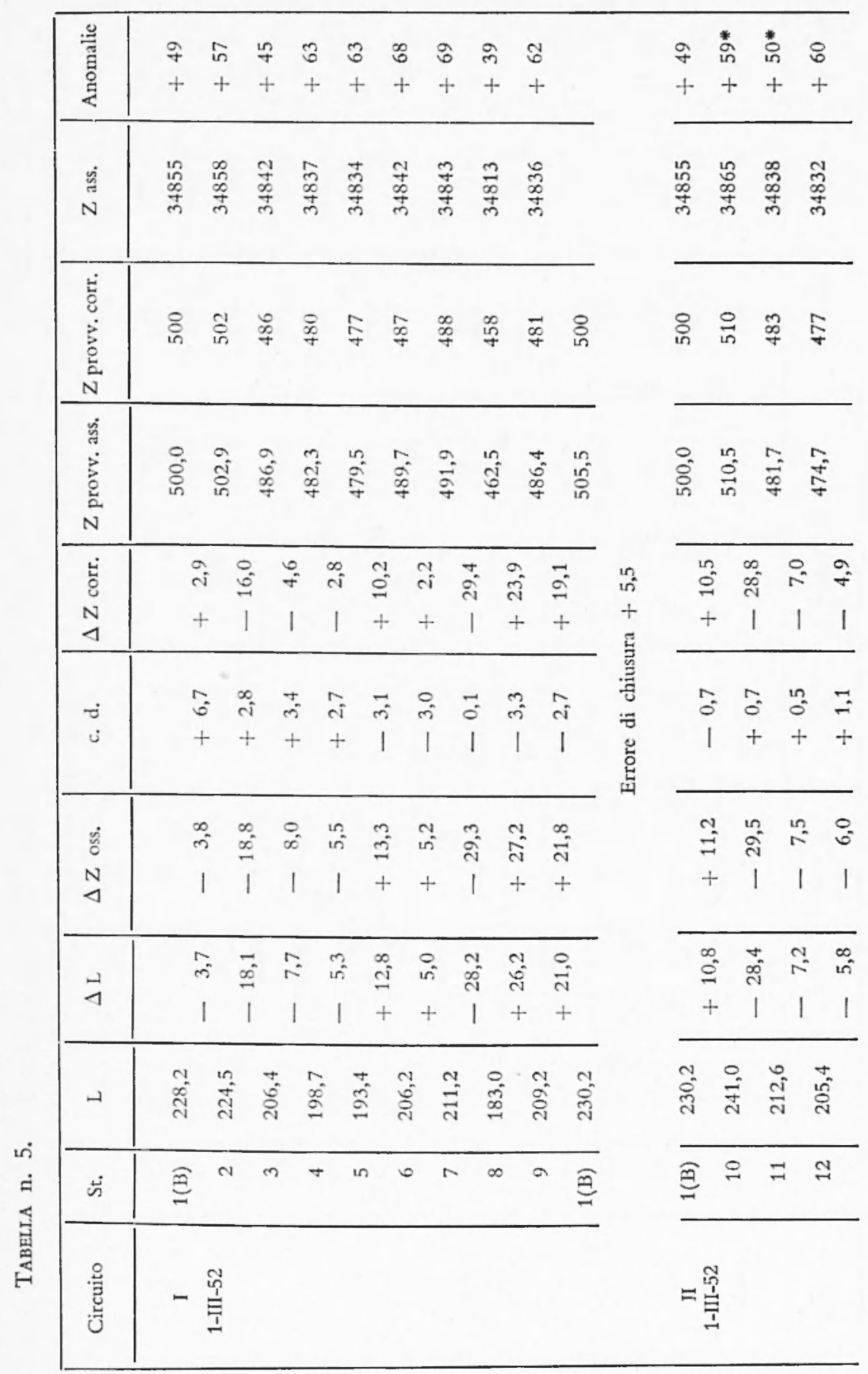




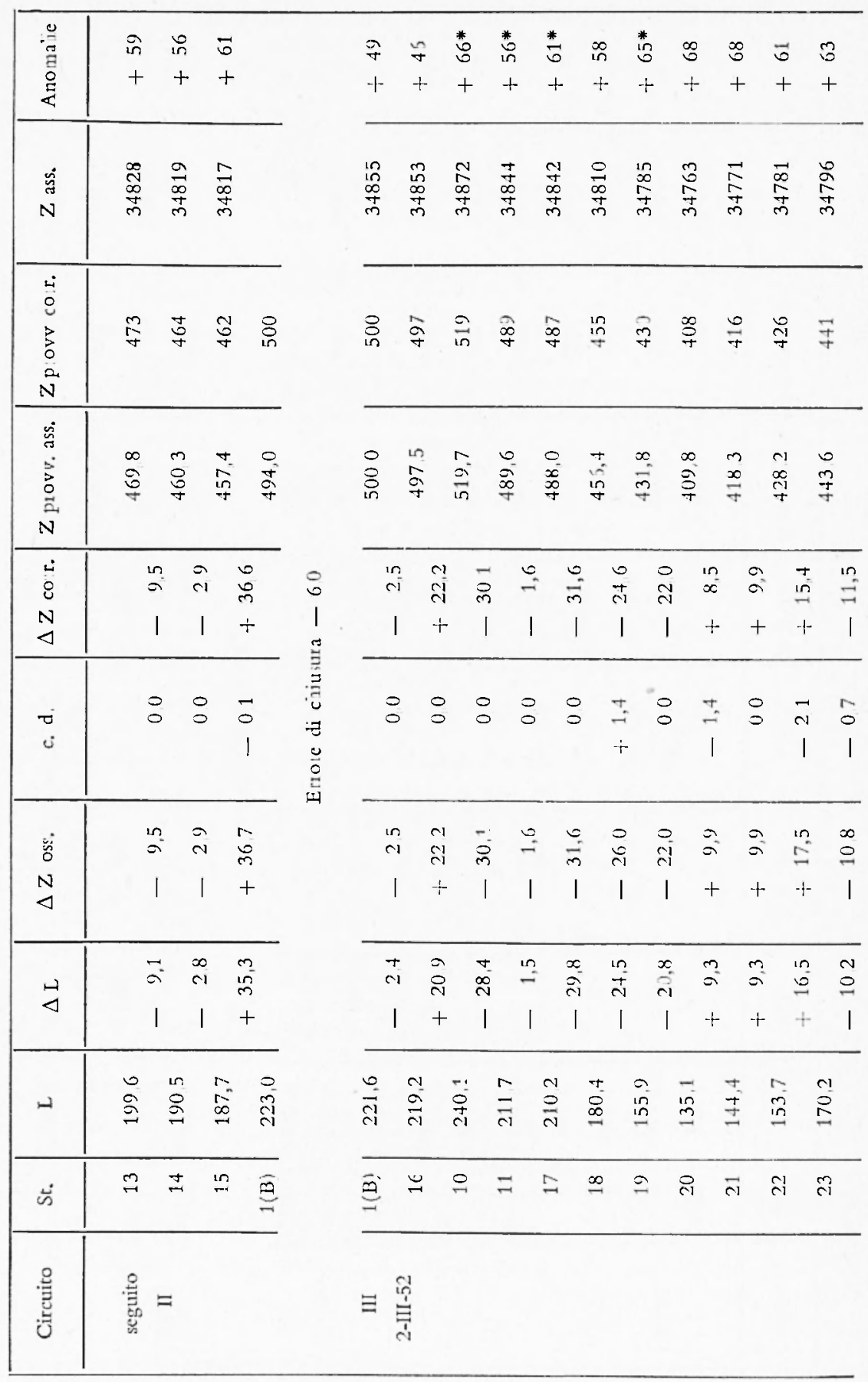




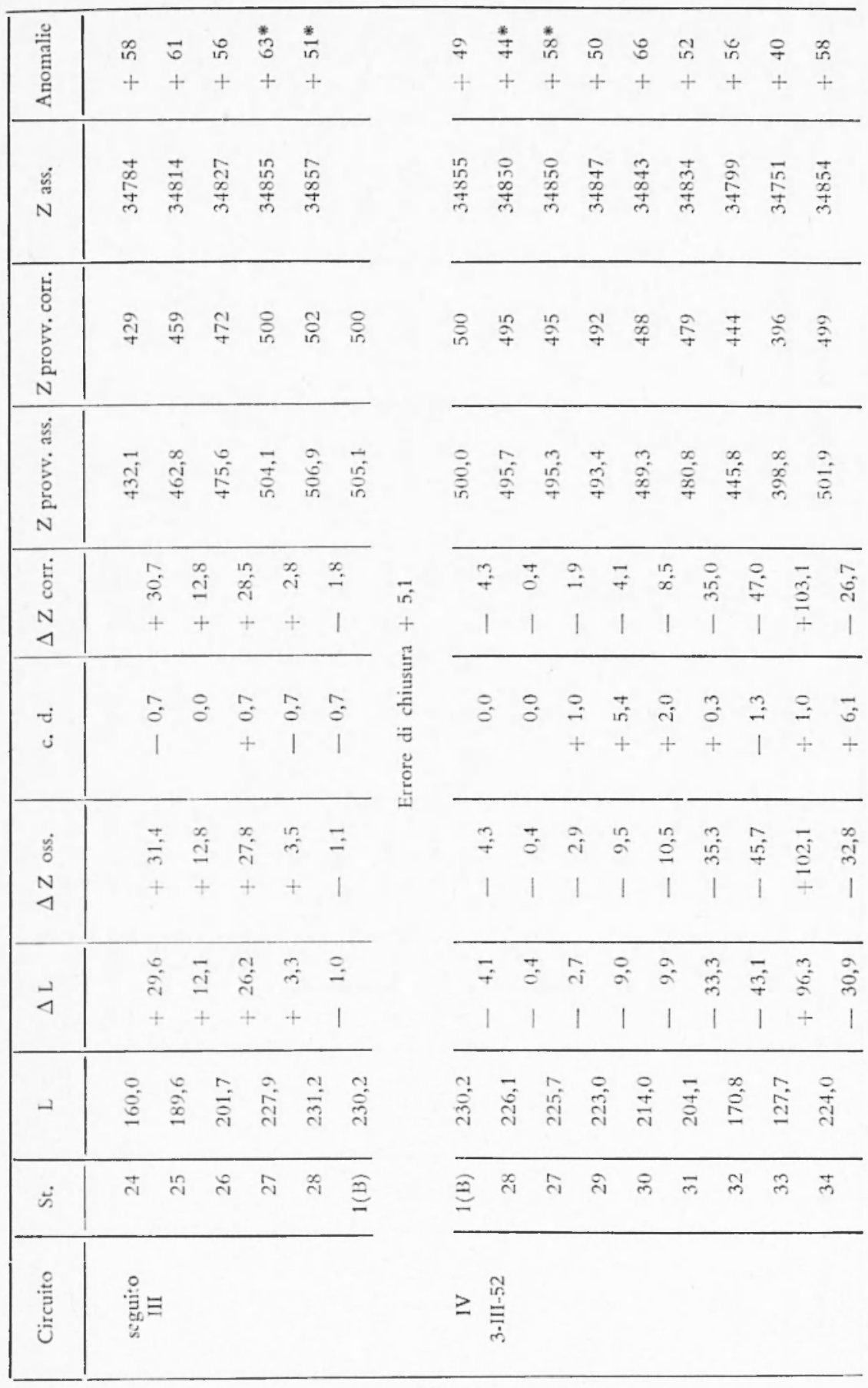




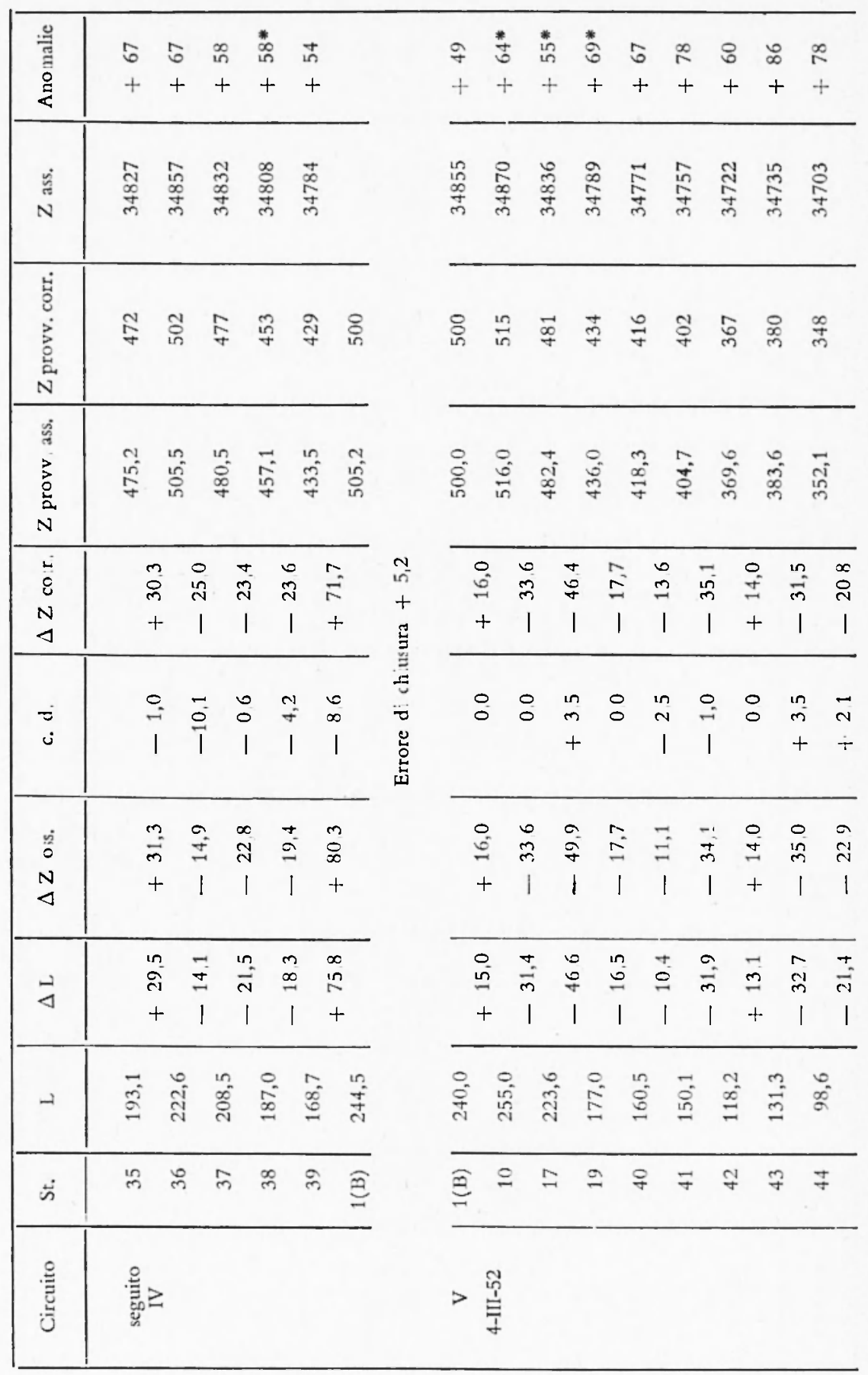




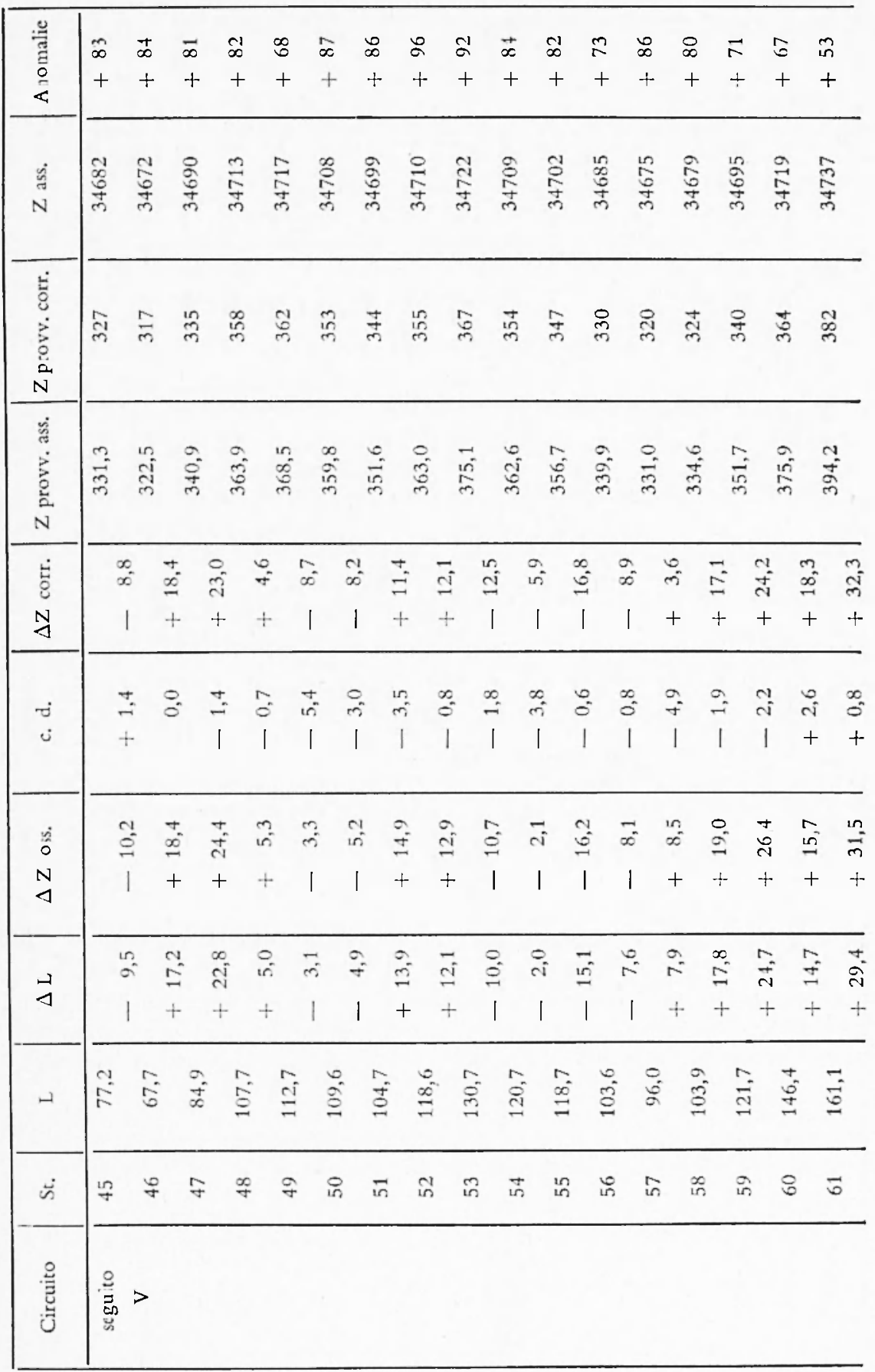




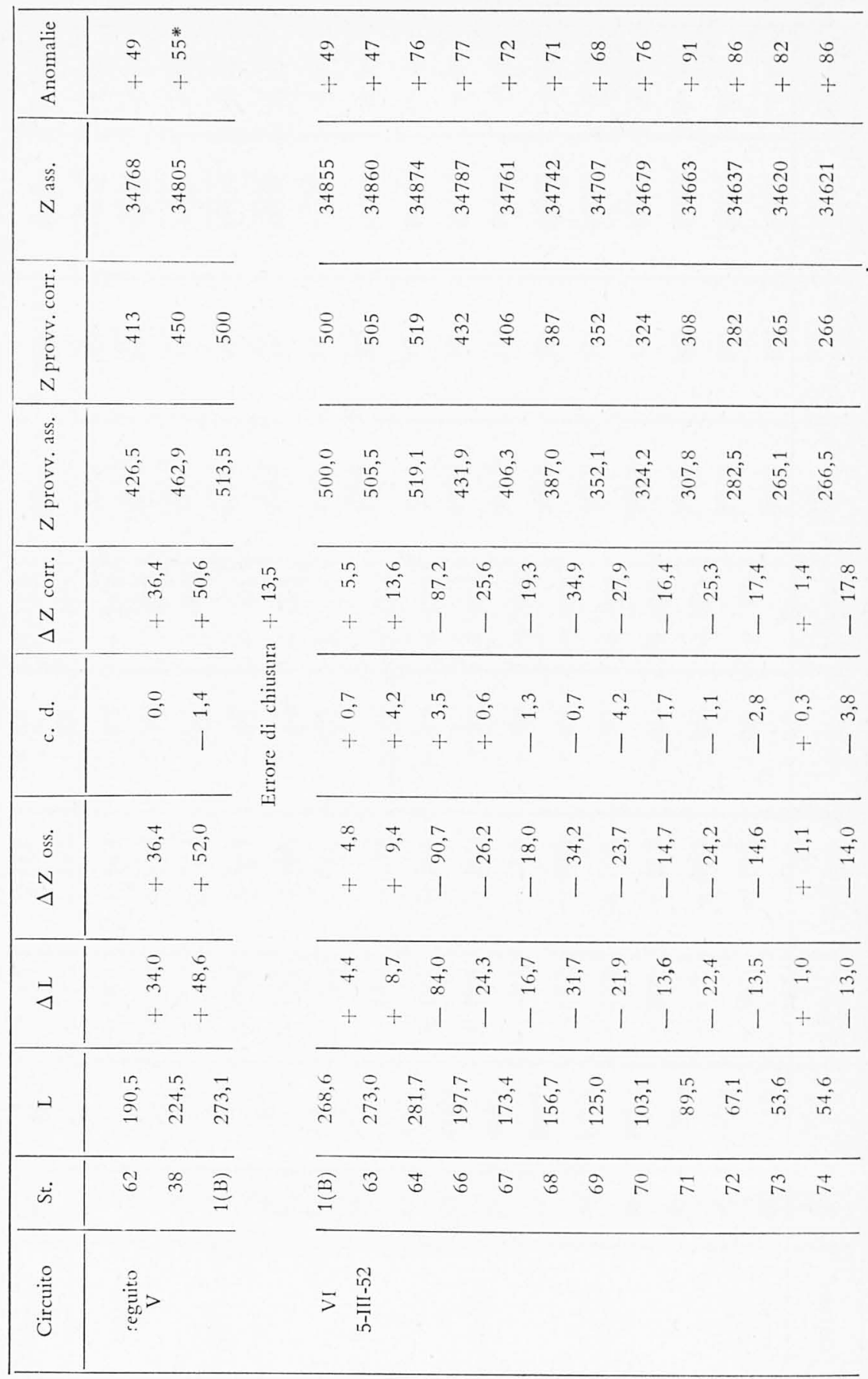




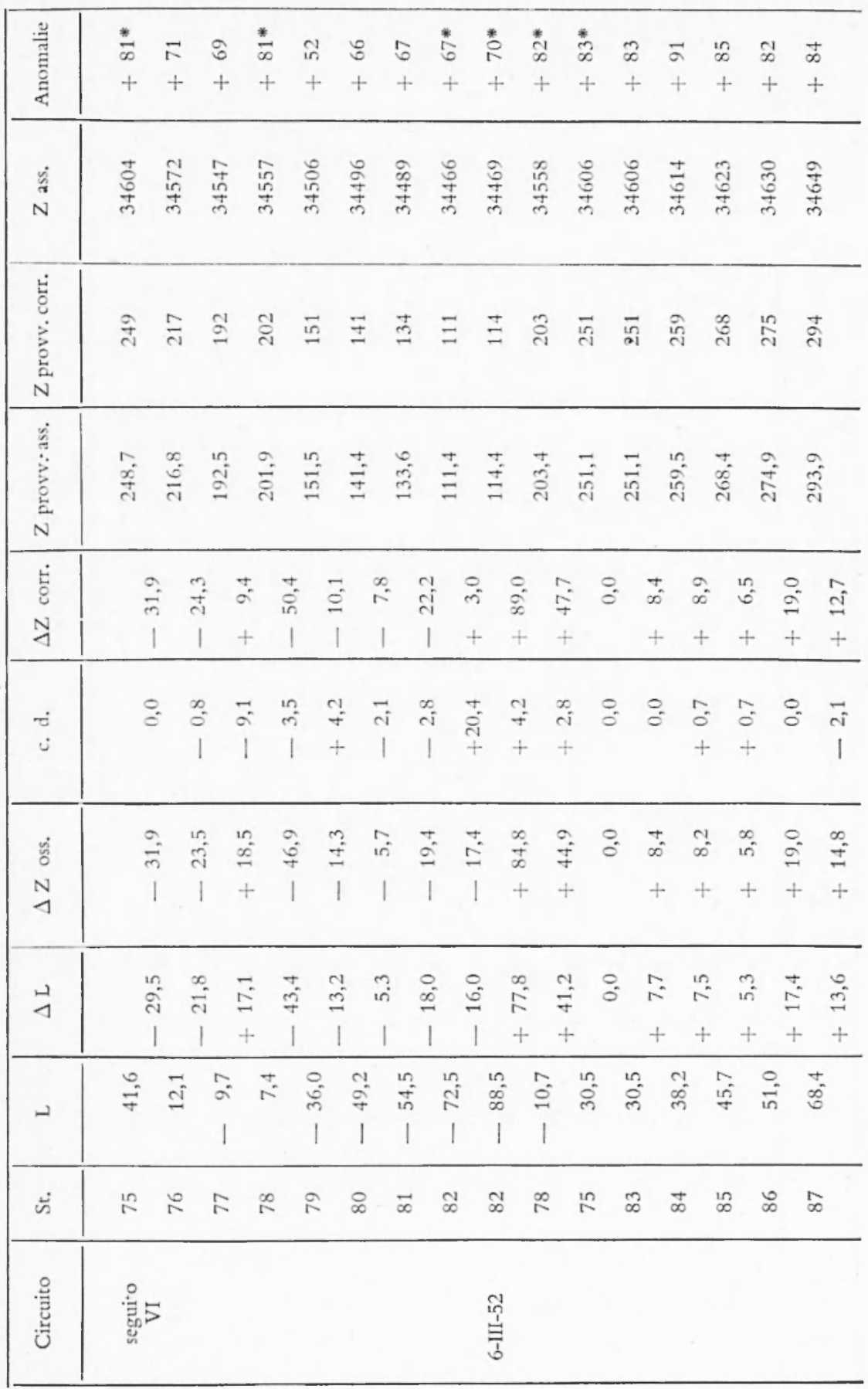




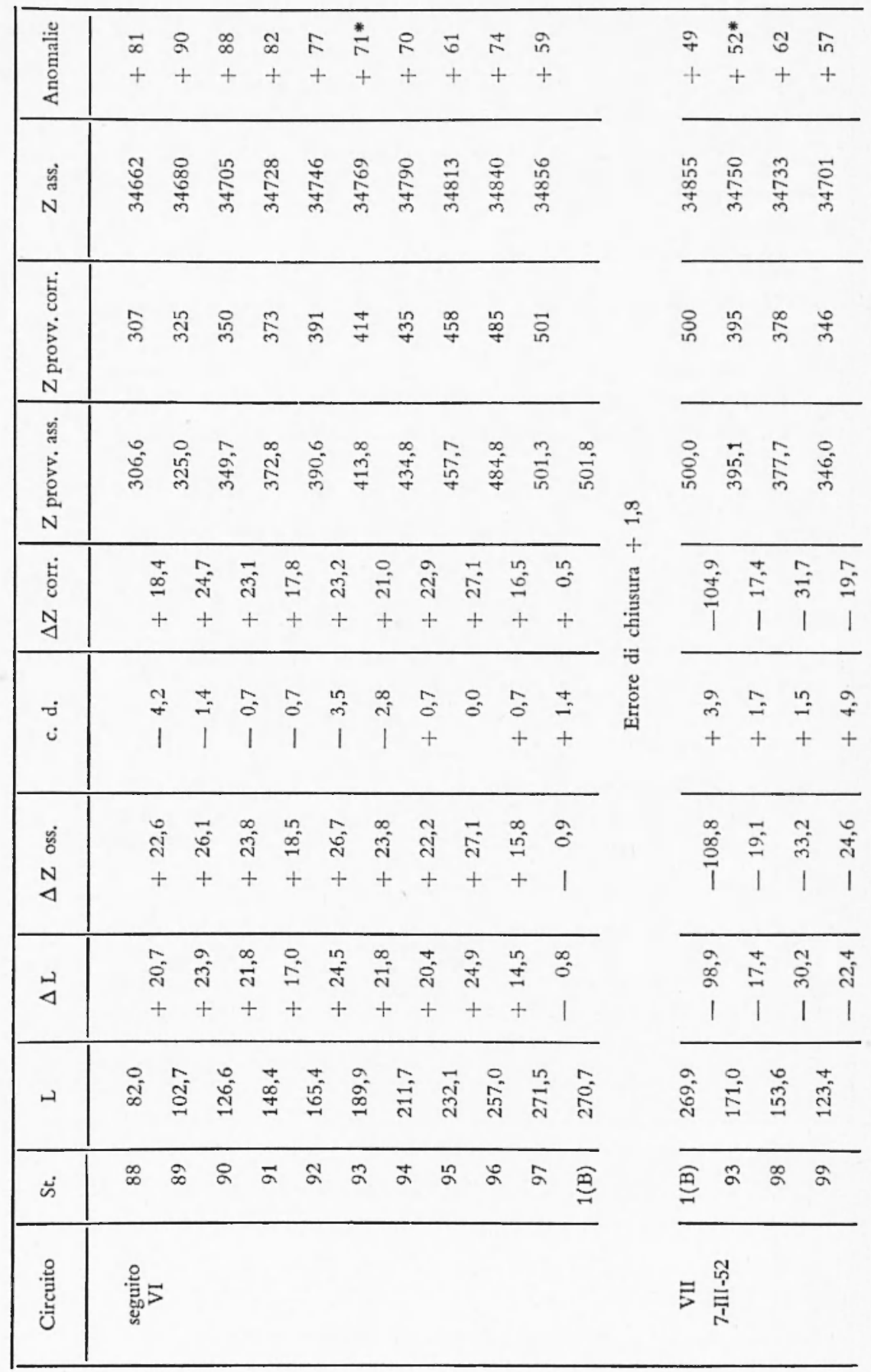




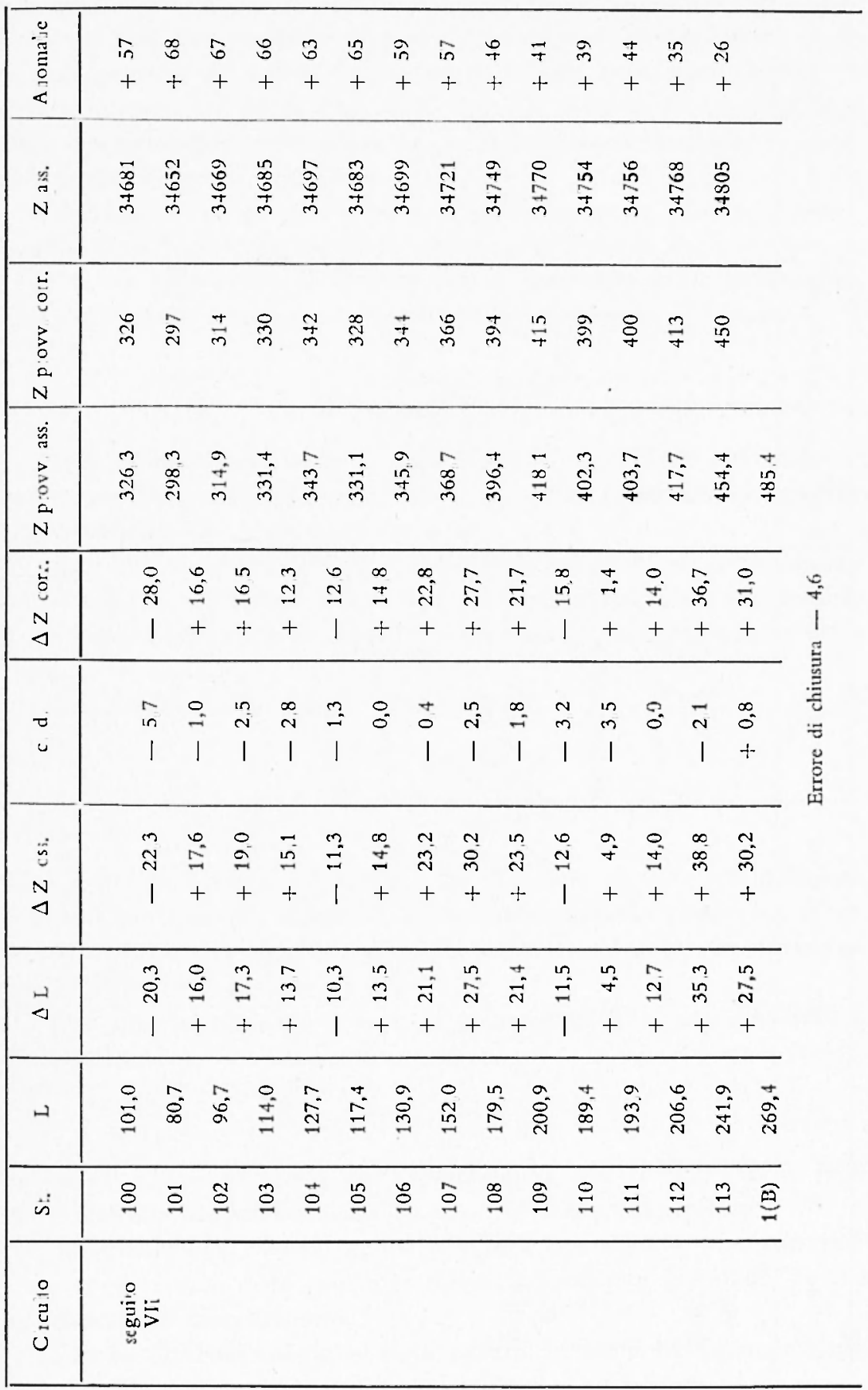




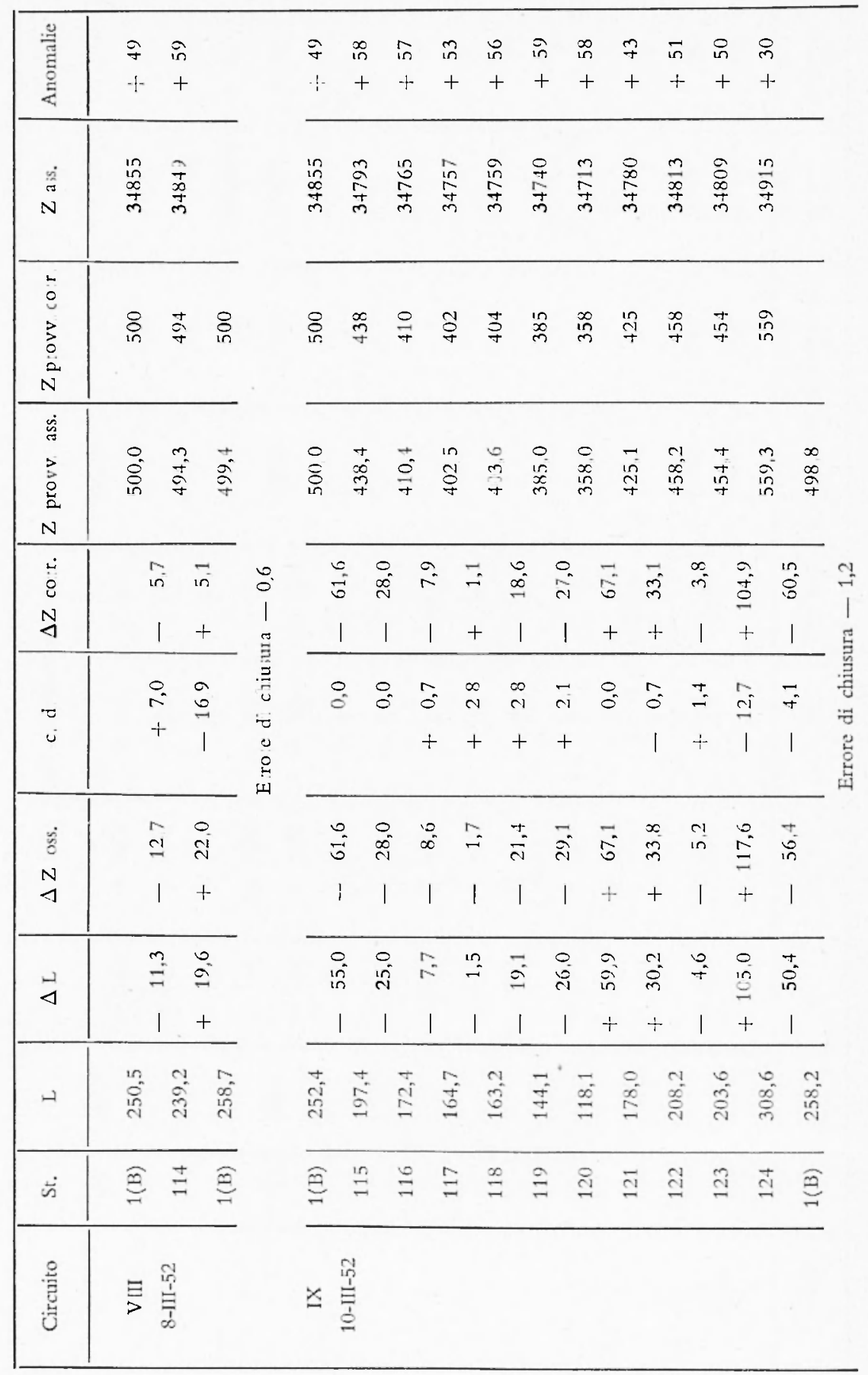


Le bilance sono state poste sempre alla stessa altezza dal suolo. In molti casi per garantire l'assenza di cause di perturbazioni accidentali proprie del punto di misura le misure sono state ripetute a diverse altezze dal suolo. Del resto, come si è detto, la "normalità " della stazione veniva controllata con la ripetizione della misura a qualche decina di metri di distanza.

Trattandosi di un rilevamento di precisione sono stati particolarwente curati l'orientamento ed il perfetto livellamento degli strumenti.

Per un variometro $I I$ l'errore che si commette nella lettura per una deviazione in azimut, $x$, rispetto all'orientamento NS esatto $\grave{e}\left({ }^{9}\right)$

$$
\Delta L=\frac{H}{\varepsilon_{\mathrm{H}}}(1-\cos \alpha)
$$

e quindi in un luogo ove $H$ è dell'ordine di $25 \cdot 10^{3} \gamma$ e per una costante di scala $\varepsilon_{H}=10 \gamma /$ div per evitare un errore superiore ad $1 \gamma$ nelle letture occorre che $\alpha$ sia inferiore a $30^{\prime}$.

Per un variometro $Z$ una deviazione rispetto all'orientamento esatto conduce ad errori delle letture compensantisi solo parzialmente nelle letture coniugate a $180^{\circ}$, e produce altresì una variazione della costante di scala.

Per l'errore nella lettura si ha infatti:

$$
\frac{\Delta I}{L}=\frac{1}{1+\frac{2 \int \varepsilon_{\mathrm{z}}}{H \cos c t}}
$$

dove $f$ per le bilance "Askania " ha il valore di $3,3 \cdot 10^{3}$ divisioni di scala: pertanto un errore di $15^{\prime}$ nell'orientamento produrrebbe un crore nelle letture agli estremi della scala di circa $l \gamma$ in un luogo ove $I I=2510^{3} \gamma$ per $\varepsilon_{2}=10 \gamma$ div.

Per quanto riguarda invece la variazione di $\varepsilon_{z}$ con l'azimut $\alpha$ avendosi :

$$
\Delta \varepsilon_{,}=\varepsilon_{\mathbf{x i}}-\varepsilon_{\mathbf{z}_{\alpha}}=-\frac{I I}{2 f} \cos \alpha
$$

un crrore di mezzo grado nell'orientamento, per $H=25 \cdot 10^{3} \gamma$, produrreble una variazione della costante di scala trascurabile.

Operando con bussole nuove e quindi con agate e punte in perfette condizioni $i$ stato possibile ridurre al minimo gli errori dovuti ad imperfetto orientamento.

Anche il livellamento $\dot{e}$ stato curato in maniera da assicurare, 
mediante un frequente controllo della rettifica delle livelle, le migliori condizioni di funzionamento delle bilance.

Durante la campagna sono state eseguite, mediante bobine di Helmholtz, varie tarature degli istrumenti i cui risultati vengono riportati nella tabella $n$. 6 .

Tab. n. 6

\begin{tabular}{|l|c|c|}
\hline Data & Costanti di scala in $\gamma /$ div \\
\cline { 2 - 3 } & $\varepsilon_{Z}$ & \multicolumn{1}{|c|}{$\varepsilon_{I 1}$} \\
\hline 1-III-52 & $10,23 \pm 0,11$ & $8,31 \pm 0,10$ \\
$2-$ III-52 & $10,57 \pm 0,09$ & $8,29 \pm 0,11$ \\
$5-$ III-52 & $10,74 \pm 0,12$ & $8,28 \pm 0,12$ \\
$8-I 1 I-52$ & $11,24 \pm 0,12$ & $8,19 \pm 0,09$ \\
& &
\end{tabular}

Oltre ai controlli della stazione base si è avuta la possibilita di una confortante conferma della attendibilità dei dati di osservazione dal confronto dei risultati delle misure in una diecina di stazioni ripetute (tab. 7).

Tab. n, 7 - Valori variometrici delle stazioni ripetute

\begin{tabular}{|c|c|c|c|c|}
\hline St. & Data & tcmp & $\begin{array}{c}\mathrm{H}_{\mathrm{c}} \\
\text { corretto }\end{array}$ & $\begin{array}{c}Z_{\mathbf{t}} \\
\text { corretto }\end{array}$ \\
\hline 10 & $\begin{array}{l}1-I I I \\
2 " \\
4\end{array}$ & $\begin{array}{l}13,5 \\
16,0 \\
14,7\end{array}$ & $\begin{array}{r}- \\
481 \\
477\end{array}$ & $\begin{array}{l}510 \\
519 \\
515\end{array}$ \\
\hline 11 & 1 & $\begin{array}{l}12,6 \\
16,2\end{array}$ & - & $\begin{array}{l}483 \\
489\end{array}$ \\
\hline 17 & $\begin{array}{l}2 " \\
4 "\end{array}$ & $\begin{array}{l}17,0 \\
15,0\end{array}$ & $\begin{array}{l}497 \\
496\end{array}$ & $\begin{array}{l}487 \\
481\end{array}$ \\
\hline 19 & $\begin{array}{l}2 \\
4\end{array}$ & $\begin{array}{l}18,6 \\
16,0\end{array}$ & $\begin{array}{l}529 \\
513\end{array}$ & $\begin{array}{l}430 \\
434\end{array}$ \\
\hline 27 & $\begin{array}{ll}2 & 4 \\
3 & 4\end{array}$ & $\begin{array}{l}19,5 \\
16,0\end{array}$ & $\begin{array}{l}513 \\
511\end{array}$ & $\begin{array}{l}500 \\
495\end{array}$ \\
\hline 28 & $\begin{array}{ll}2 & \text { " } \\
3 & \text { " }\end{array}$ & $\begin{array}{l}20,5 \\
16,0\end{array}$ & $\begin{array}{l}509 \\
497\end{array}$ & $\begin{array}{l}502 \\
495\end{array}$ \\
\hline 38 & $\begin{array}{l}3 \text { " } \\
4 "\end{array}$ & $\begin{array}{l}21,5 \\
12,0\end{array}$ & $\begin{array}{l}521 \\
522\end{array}$ & $\begin{array}{l}453 \\
450\end{array}$ \\
\hline 75 & $\begin{array}{ll}5 & 4 \\
6 & 4\end{array}$ & $\begin{array}{l}20,0 \\
18,2\end{array}$ & $\begin{array}{l}627 \\
626\end{array}$ & $\begin{array}{l}249 \\
251\end{array}$ \\
\hline 78 & $\begin{array}{ll}5 & 4 \\
6 & 4\end{array}$ & $\begin{array}{l}19,0 \\
17,7\end{array}$ & $\begin{array}{l}670 \\
671\end{array}$ & $\begin{array}{l}202 \\
203\end{array}$ \\
\hline 82 & $\begin{array}{l}5 \text { " } \\
6 "\end{array}$ & $\begin{array}{l}18,0 \\
18,5\end{array}$ & $\begin{array}{l}756 \\
747\end{array}$ & $\begin{array}{l}111 \\
114\end{array}$ \\
\hline 93 & 6 & $\begin{array}{l}20,0 \\
17,2\end{array}$ & $\begin{array}{l}533 \\
541\end{array}$ & $\begin{array}{l}414 \\
395\end{array}$ \\
\hline
\end{tabular}


Correzioni delle misure.

a) Correzione per la temperatura. - Come si è potuto constatare in precedenti appositi controlli e come appare nella tabella $n$. 1 contenente le caratteristiche strumentali, le bilance magnetiche adoperate per il rilevamento erano fornite di equipargi compensati per le variazioni di temperatura contenute entro i limiti di $10^{\circ}$ e $45^{\circ} \mathrm{C}$, per valori di $I I=0,2515 \mathrm{\Gamma}$ e di $Z=0,3500 \Gamma$. Dato che $\left({ }^{10}\right)$ tale compensazione sussiste anche per variazioni del campo di qualche migliaio di $i$ attorno ai valori di taratura, avendo operato praticamente entro i suddetti limiti di temperatura e avendo anche sempre avuto cura di evitare cambiamenti bruschi della temperatura degli strumenti, non ¿̀ stato necessario apportare alcuna correzione per questa causa.

b) Correzioni per la variazione diurna.

1) Stazione registratrice da campo. In considerazione del fatto che si sarchbe dovuto operare in una zona magneticamente " normale $)$ e che quindi avrebbero dovuto essere messe in evidenza piccole differenze dei valori di entrambe le componenti $H$ e $Z$ fra punto e punto della zona rilevata, si è ritenuto necessario avere un controllo minuto e rigoroso della variazione diurna. A tal fine è stata allestita, oltre al complesso di variografi “Ruska » dell'Osservatorio di Gibilmanna, una stazione di registrazione da campo con bilance registratrici Askania a cellula fotoelettrica, una per la componente $H$ e l'altra per la componente $Z$.

L'impianto di detta stazione da campo è stato eseguito al piano terreno di una casa colonica temporaneamente non abitata (casa Flaccomioj in prossimita della stazione base, precisamente nel punto di coordinate:

$$
\varphi=38^{\circ} 01^{\prime} 28^{\prime \prime} N \quad \lambda=1^{\circ} 34^{\prime} 29^{\prime \prime} E \text { (M. Mario) }
$$

Il locale, ben protetto dalle variazioni di temperatura, era abbastanza lontano dalla strada e da cause di perturbazioni accidentali.

È stata eseguita all'inizio ed alla fine delle operazioni di campagna la taratura delle bilance registratrici mediante la bobina di Helmholtz: i risultati sono riportati nella tabella $n .8$.

La stazione di registrazione ha funzionato regolarmente per tutta la durata della campagna ed i diagrammi ottenuti sono risultati in buon accordo con quelli delle registrazioni fotografiche dei variografi 
"Ruska » situati a qualche chilometro di distanza. Notiamo tuttavia che le registratrici a cellula fotoelettrica non sempre danno garanzia della assoluta fedeltà delle registrazioni.

2) Stazione variografica di Gihilmanna. Presso l'Osservatorio di Gihilmanna che si trova a $1000 \mathrm{~m}$ s.l.m. in prossimita di Pizzo S. Angelo a sud di Cefalù, in un padiglione sotterranco si trovava già in-

Tab. n. 8

\begin{tabular}{|c|c|c|}
\hline Data & \multicolumn{2}{|c|}{ Costanti di scala in $\gamma /$ div } \\
\cline { 3 - 3 } & $\varepsilon_{Z}$ & $\varepsilon_{I I}$ \\
\hline $29-I I-52$ & $1,29 \pm 0,03$ & $1,12 \pm 0,02$ \\
$11-$ III-52 & $1,25 \pm 0,02$ & $1,09 \pm 0,03$ \\
& & \\
\end{tabular}

Tab. 11. 9

\begin{tabular}{|c|c|c|c|} 
Data & $\begin{array}{c}\varepsilon_{\mathrm{I}} \\
\gamma^{\prime} \mathrm{mm} .\end{array}$ & $\begin{array}{c}\varepsilon_{\mathrm{H}} \\
\gamma / \mathrm{mm} .\end{array}$ & $\begin{array}{c}\varepsilon_{\%} \\
\gamma / \mathrm{mm} .\end{array}$ \\
\hline $1-\mathrm{III}-52$ & $8,12 \pm 0,06$ & $4,91 \pm 0,07$ & $7,05 \pm 0,05$ \\
& & &
\end{tabular}

stallato e funzionante da circa un anno un complesso variografico "Ruska " da osservatorio per gli elementi $D, H$ e $Z$.

Le registrazioni fotografiche ottenute con questo complesso variografico hanno fornito le migliori registrazioni per la riduzione dei dati di osservazione.

I dati di taratura dei variografi “ Ruska » vengono riportati nella tabella n. 9.

I grafici dell'Osservatorio di Gihilmanna e della stazione registratrice da campo hanno consentito una determinazione sufficientemente precisa delle riduzioni delle misure quale è stata necessaria data la esiguita delle anomalie della zona rilevata e soprattutto data la irregolarità dell'andamento della variazione diurna durante diversi giorni dello svolgimento della campagna.

Abliamo ritenuto opportuno riportare nei grafici delle figure 1 e 2 l'andamento della variazione diurna di $H$ e $Z$ durante i giorni delle operazioni di campagna.

Verso le $13^{\text {li }}$ del giorno 3 marzo ha inizio una tempesta magnetica di una certa intensiti, che si è prolungata praticamente per tutta la durata della campagna.

La tempestiva conoscenza di questo fatto ci ̀̀ stata utile per eseguire le misure con la massima accuratezza, specialmente nella deter* 
minazione degli istanti delle letture, per evitare un dannoso rinvio delle operazioni di campanna.

Sovrapposto alla consueta diminuzione del valore di $\boldsymbol{H}$ ed all'aumento di $Z$, la tempesta presenta un rapido susseguirsi di « baie » positive e negative specialmente della componente $H$, che talvolta hanno prodotto variazioni fino ad $80 y$ in mezz'ora. La massima intensità della perturbazione si ̀̀ avuta nel pomeriggrio del giorno 5 marzo e nella mattinata del 6 , come si può vedere anche dalla tabella $n$. 10 in cui

Tab. n. 10 - Indici Kp.

\begin{tabular}{|c|c|c|c|c|c|c|c|c|}
\hline $\begin{array}{c}\text { Ore } \\
\text { T.M.C. }\end{array}$ & ()-3 & $3-6$ & $6-9$ & $9-12$ & $12-15$ & $15-18$ & $18-21$ & $21-24$ \\
\hline $1-111-52$ & 4 & 4 & 3 & 3 & 4 & 3 & 1 & 1 \\
\hline $2 "$ & 1 & 3 & 1 & 2 & 1 & 1 & 1 & 1 \\
\hline 3 & 0 & 1 & 2 & 2 & 6 & 5 & 6 & 7 \\
\hline 4 & 6 & 6 & 6 & 6 & 5 & 5 & 6 & 5 \\
\hline $5 "$ & 6 & 7 & 5 & 6 & 6 & 6 & 6 & 7 \\
\hline $6 \pi$ & 8 & 7 & 6 & 5 & 4 & 4 & 5 & 5 \\
\hline 7 & 4 & 5 & 3 & 6 & 5 & 6 & 5 & 7 \\
\hline $8 "$ & 6 & 5 & 4 & 5 & 5 & 6 & 6 & 5 \\
\hline 10 & 4 & + & 5 & 4 & 4 & 6 & 5 & 5 \\
\hline
\end{tabular}

abbiamo riportato gli indici $K$ planetari (11). In tali condizioni sarebbe stato evidentemente impossibile applicare la correzione per la variazione diurna basandosi sulle curve medie normali o quanto meno su registrazioni men che precise del c.m.t. - E stato invece necessarto riportare sulle registrazioni gli istanti esatti delle stazioni eseguite in ogni circuito.

La regolarita di funzionamento delle due stazioni di registrazione, specialmente quella dell’Osservatorio di Gibilmanna, e la precisione dei diagrammi ottenuti hanno permesso di determinare le correzioni con la dovuta accuratezza.

La attendibilità dei risultati può vedersi soprattutto nella concordanza dei valori delle stazioni ripetute riportati nella tabella $n$. 7 in cui i valori sono già corretti per l'errore di chiusura di cui si parla più sotto.

Si può notare anzi, per esempio, che per le stazioni n. 75,78 e 82 in cui le misure sono state eseguite proprio nel periodo della massima intensità della perturhazione, l'accordo è molto soddisfacente. 


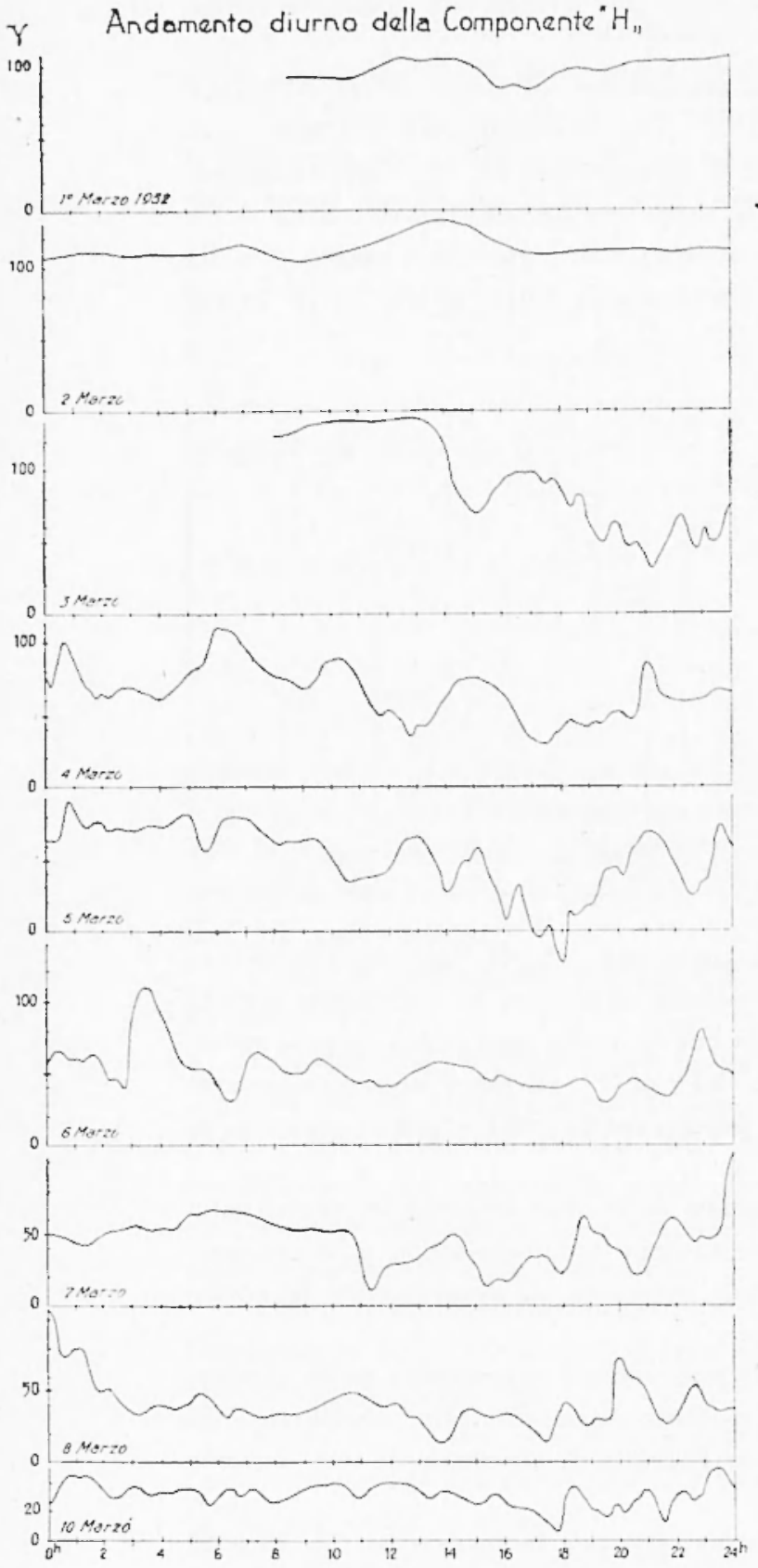

Fig. 1

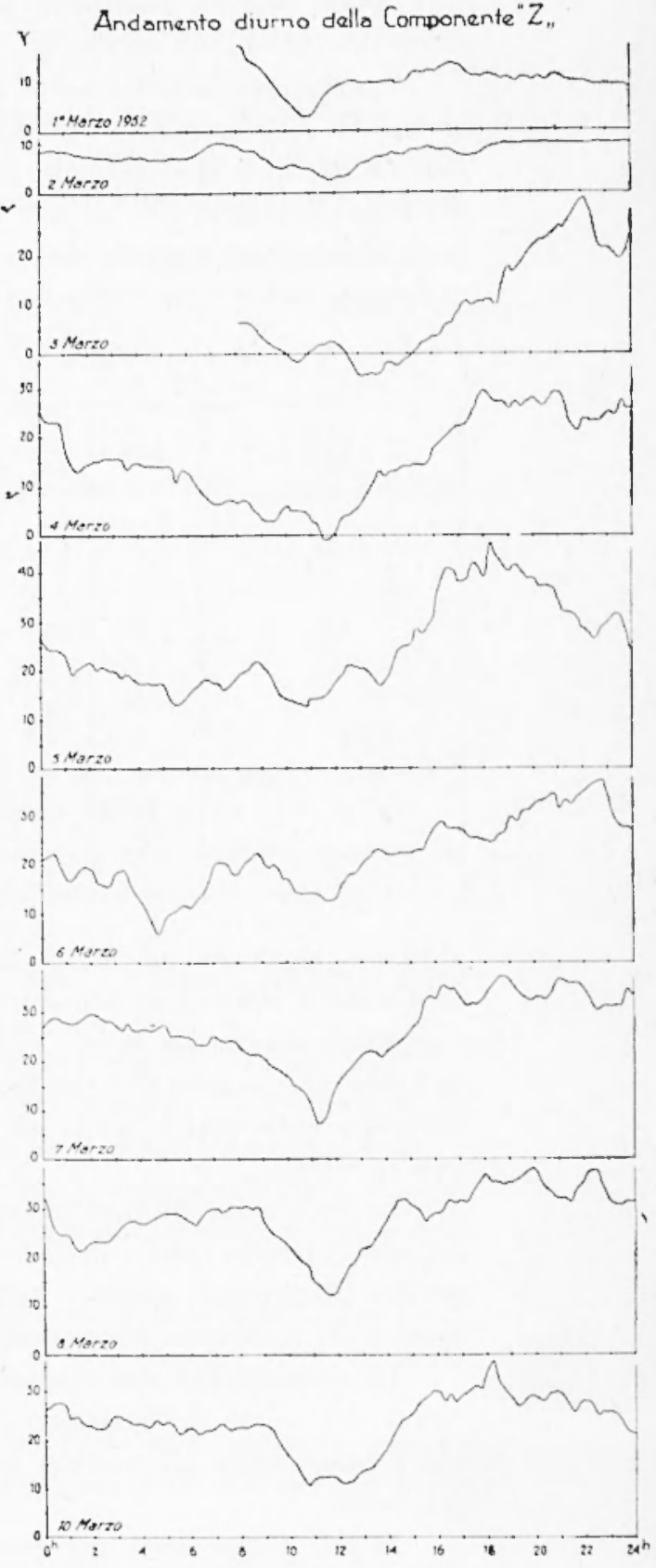

Fig. 2 
c) Correzioni di chiusura. - Gli errori di chiusura, differenza fra i valori delle misure iniziali e finali alla stazione base per ogni circuito, sono maggiori per la componente $I$ che per la $Z$.

Nella tabella n. 11 vengono riportati gli errori di chiusura ed il numero di stazioni per ciascun circuito.

Tab. n. 11

\begin{tabular}{|c|c|c|c|}
\hline Circ. & $n$ & $c_{H}$ & $c_{Z}$ \\
\hline 1 & 8 & $+10,8$ & - \\
\hline & 10 & $\rightarrow$ & $+5,5$ \\
\hline 2 & $\begin{array}{l}6 \\
8\end{array}$ & $-18,7$ & $--^{6} .0$ \\
\hline 3 & 17 & $-7,1$ & $\begin{array}{r}5,1 \\
+\quad 5\end{array}$ \\
\hline 4 & 15 & $+23,2$ & $+5,2$ \\
\hline 5 & 29 & $+19,4$ & $+13,5$ \\
\hline 6 & 39 & $+16,9$ & \\
\hline 7 & 19 & $+6,5$ & $-4,6$ \\
\hline 8 & 3 & $+1,4$ & \\
\hline 9 & 12 & $+4,3$ & $-1,2$ \\
\hline
\end{tabular}

Gli errori di chiusura, in considerazione del fatto che le misure sono state cseguite in condizioni magneticamente perturbate, sono relativamente piccoli, comunque sono stati equipartiti $\left({ }^{12}\right)$ fra le stazioni di ciascun circuito applicando alle misure la correzione:

$$
\frac{t_{2}-t_{1}}{n} m
$$

dove $t_{1}$ e $t_{2}$ sono i valori iniziali e finali, espressi in $\gamma$, alla stazione base, $n$ il numero di stazioni nel circuito e $m$ l'ordine delle stazioni a partire dalla stazione base.

Precisione del rilevamento. -- Gli errori medi di ogni singola stazione sono stati calcolati in base agli errori di chiusura dei diversi circuiti dando a ciascun valore un peso inversamente proporzionale al numero di stazioni comprese nel corrispondente circuito

$$
m_{\mathrm{II}}= \pm \sqrt{\frac{\left|p_{\mathrm{H}}^{2}\right|}{2[p]}}=-8,5 \gamma \quad m_{\mathrm{z}}= \pm \sqrt{\frac{\left[p_{\mathrm{z}}\right]}{2[p]}}= \pm 4,0 \cdot \gamma
$$

Tenendo conto oltre agli errori di chiusura alla stazione base anche delle stazioni ripetute (tal). n. 7) si ha invece:

$$
m_{\mathrm{H}}^{\prime}= \pm \sqrt{\frac{\left[\rho_{\mathrm{H}} \rho_{\mathrm{H}}\right]}{2 n}}= \pm 7,5 \gamma \quad m_{\mathrm{Z}}^{\prime}= \pm \sqrt{\frac{\left[e_{\mathrm{Z}} e_{\mathrm{Z}}\right]}{2 n}}= \pm 4,4 \gamma
$$


Nel calcolo delle isoanomale sono stati attribuiti alle stazioni ripetute i valori medi di $H$ e $Z$ osservati. Pur operando con strumenti in perfetta efficienza, usando tutti gli accorgimenti opportuni e applicando rigorosamente le norme proprie di questo genere di misure, si vede che la precisione di ogni stazione è risultata dell'ordine di $+\gamma$ per la componente $Z$ e 8 r per la componente $H$.

Detti valori sono da ritenersi soddisfacenti per quanto non molto piccoli.

Valori assoluti. - Durante una campagna magnetica condotta nel marzo 1954 (13) abbiamo eseguito misure mannetiche assolute degli elementi $D, H$ e $I$ in 4 punti coincidenti con altreltante stazioni assolute a suo tempo battute dall'Istituto Geografico Militare, comprese nella zona del rilievo del $\mathrm{II}^{\circ}$ ordine.

I dati ottenuti, $H$ e $Z$, ridlotti alla data del 28 marzo 1954 sono riportati nella tabella $n .12$ a fianco dei ralori provvisori relativi $h$ e $z$ riferiti al valore convenzionale 500 della stazione base.

Tab. 11. 12

\begin{tabular}{|c|c|c|c|c|c|c|c|c|}
\hline \multirow{2}{*}{ St. } & \multicolumn{2}{|c|}{$\begin{array}{c}\text { Valori assoluti } \\
\text { osservati al } 1954,25\end{array}$} & \multicolumn{2}{|c|}{ Valori variometrici } & \multicolumn{2}{|c|}{ Valori compensati } & \multirow{2}{*}{$\mathrm{H}-\mathrm{H}_{\mathrm{r}}$} & \multirow{2}{*}{$\mathrm{Z}-\mathrm{Z}_{\mathrm{c}}$} \\
\hline & $\mathrm{H}$ & $\mathrm{Z}$ & $\mathrm{H}_{\mathrm{v}}$ & $Z_{v}$ & $\mathrm{H}_{\mathrm{c}}$ & $\mathrm{Z}_{c}$ & & \\
\hline 82 & 26079 & 34446 & 751 & 113 & 26073 & 34469 & +6 & -23 \\
\hline 57 & 25866 & - & 550 & - & 25872 & - & -6 & - \\
\hline 111 & 25842 & 34768 & 511 & 400 & 25833 & 34756 & +9 & +12 \\
\hline 124 & 25757 & 34927 & 445 & 559 & 25767 & 34915 & -10 & +12 \\
\hline
\end{tabular}

I valori assoluti di $H$ e $Z$ sono stati corretti mediante una compensazione tra misure assolute e misure relative ottenuta imponendo le seguenti due condizioni:

$$
\begin{gathered}
\sum_{i}^{\mathrm{n}}\left(T ;-x_{\mathrm{i}}\right)=0 \\
x_{\mathrm{i}}-x_{\mathrm{i}+1}=t_{\mathrm{i}}-t_{\mathrm{i}+1}
\end{gathered}
$$

dove le $T_{1}$ sono i valori assoluti osservati della componente considerata nelle diverse stazioni, le $t_{1}$ sono $i$ corrispondenti valori relativi provvisori e le $x_{i}$ i valori assoluti ottenuti dalla compensazione. La 
tabella n. 12 contiene i valori usati nel calcolo della compensazione.

In detta tabella manca il valore della $Z$ nella stazione n. 57 (Sperlinga) dove non si è potuta misurare la $I$ a causa delle condizioni meteorologiche avverse.

Ottenuti i quattro valori compensati, sono stati calcolati, in base ai valori relativi provvisori, $i$ valori assoluti in tutte le altre stazioni, ridotti al $1954,25$.

Campo normale ed anomalie regionali. - Nel lavoro sopra citato $\left({ }^{13}\right) \dot{e}$ stato calcolato il campo normale per le componenti $H$ e $Z$ della Sicilia; esso è dato mediante le seguenti espressioni di $\mathrm{II}^{\circ}$ grado in $\varphi$ e $\lambda$ :

$I_{\mathrm{n}}=27104,1-20,536 \varphi-1,687 \lambda+0,05586 \varphi^{2}+0,00191 \lambda^{2}+0,01400 \varphi \lambda$

$Z_{11}=33708,4-9,444 \varphi-0,800 \lambda+0,10170 \varphi^{2}+0,00503 \lambda^{2}-0,00147 \rho \lambda$

Le [1] sono state ricavate dai valori $H$ e $Z$ ridotti al 1935,0 dedotti dalle misure eseguite dall'Istituto Geografico Militare in n. 81 stazioni, avendo escluse tutte le stazioni anomale. Nelle [1] $\varphi$ e $\lambda$ sono misurate in primi; $\varphi$ è contata a partire da $36^{\circ}$ di lat. $N$ e $\lambda$ dal meridiano di Monte Mario.

Per avere il campo normale al 1954,25 della regione da noi rilevata, ai valori del campo normale ottenuti mediante le [1] per le n. 124 stazioni del secondo ordine abbiamo apportato la correzione per la variazione secolare.

Come i descritto nel sopra citato lavoro, mediante le misure magnetiche assolute eseguite nel mese di marzo 1954 in quattro stazioni dell'Istituto Geografico Militare comprese nella zona da noi rilevata abbiamo computato un valore della variazione secolare molto attendibile per gli anni compresi fra il 1935 e il 1954 sia per la componente $H$ come per la $Z$; detti valori possono ritenersi validi per tutta la zona in cui abbiamo eseguito le misure relative e sono rispettivamente:

$$
\frac{\Delta H}{\Delta t}=+17,8 \% / a \quad \frac{\Delta Z}{\Delta t}=+41,2 \gamma / a
$$

Le differenze fra $i$ valori assoluti delle varie stazioni e $i$ valori normali calcolati mediante le [1] corrette per la variazione secolare danno le anomalie regionali.

Nelle carte n. 3 e n. 4 sono state riportate rispettivamente le iso- 
dinamiche orizzontali e verticali, mentre le carte n. 5 e 6 danno rispettivamente l'andamento delle isoanomale orizzontali e verticali.

Naturalmente le isomonale regionali per $H$ e $Z$, calcolate nel s:odo anzidetto, dipendono dal particolare campo normale prescelto.

II calcolo e l'uso del campo normale dato dalle [1] è giustificato dalle seguenti ragioni:

a) esso si basa sui dati magnetici più recenti e sistematici:

b) è dedotto da valori del campo riferentisi alla sola Sicilia in modo da evitare l'influenza di caratteristiche magnetiche di altre regioni;

c) i valori del campo da cui è tratto non sono anomali, sono abbastanza numerosi e uniformemente distribuiti su tutta la rerrione.

Gli errori medi unitari di $H$ e $Z$ ricavati dalle relazioni [1] sono rispettivamente $\left({ }^{13}\right)$.

$$
\mu_{\mathrm{H}}=-23,9 \gamma \quad \mu_{\gamma}=+48,7 \gamma
$$

cioè dello stesso ordine di grandezza dell'errore di cui sono aflette le misure di $H$ effettuate dall'I.G.M. e dell'errore di cui risulta affetto $Z$ dedot to dalle misure di $H$ e di $I\left({ }^{4}\right)$.

Le anomalie calcolate nel modo sopradetto e riportate nelle tabelle n. 5 e n. 6 risultano in generale piuttosto piccole e comparabili con gli errori medi di $I_{n}$ e $Z_{n}$; tuttavia il fatto che esse mostrano generalmente un sistematico andamento nella distribuzione spaziale induce a concludere positivamente per un loro significato obbiettivo.

E da osservare però che per la componente $Z$ le anomalie risultano sensibilmente più marcate che per la $H$.

Le anomalie più notevoli sono le seguenti:

a) una anomalia positiva nella $Z$ (fino a $96 y$ ) nella zona tra Petralia e Gangi;

b) una anomalia negativa nella componente $H$ (fino a -42 ) nella zona di Nicosia.

Da quanto sopra si i detto, pur ri-ultando obbiettiva l'esistenza delle piccole anomalic calcolate mediante il criterio sopra esposto, ¿ da auspicare però un miglioramento della precisione delle misure magnetiche assolute del $1^{\circ}$ ordine, effettuando nuove osservazioni con strumenti più adatti a tale scopo quali possono essere quelli a torsione, sia per la componente orizzontale (HTM) sia per la componente verticale $(Z T M)$.

Roma - Istituto Nazionnle di Geofisica - Aprile 1954. 


\section{RIASSUNTO}

Nel quadro di un rilevamento geofisico della Sicilia, predisposto dall'Istituto Nazionale di Geofisica, è stato iniziato il lavoro di rilevamento magnetico di una vasta zona della parte centro-settentrionale dell'isola. La scelta di questa parte dolla Sicilia, già nota come non anomala, è stata motivata dalla opportunità di conoscere meglio lo caralleristiche magnetiche della zona comprendente l'osservatorio geofisico di Gibilmanna. Il territorio rilevato copre un'area di $3.000 \mathrm{kmq}$ circa.

Vengono descritte le modalitá del lavoro di campagna ed $i$ mezzi adoperati per lo misure assolute e relative effettuate. Vengono descritti ed esposti criticamente $i$ procedimenti per l'elaborazione dei dati di osservazione.

Le anomalie regionali della zona rilevata sono state calcolate per differenza dai valori del "campo normale" per le due componenti II $\mathrm{Z}$ calcoluti con $i$ duti di osservazione, ridotti al 1935,0, relativi a 125 stażioni assolute eseguite dall'Istituto Geografico Militare.

Le anomalie più notrevoli sono:

a) una anomalia positiva della $\mathrm{Z}$ (fino a $96 \%$ ) tra Petralia $e$ Gangi

b) una anomalia negativa della $H$ (fino a $-42 \gamma$ ) nella zona di Nicosin.

\section{SLMMARY}

Within the outline of a geophysical survey of Sicily, arranged by the Italian National Institute of Geophysics, a magnetic survey of a wide area of North-Central Sicily was carried out.

The choice of this part of Sicily, already noted as being not anomal, was motivated by the opportunity to obtuin better knowledge of the magnetic characteristics of the area comprising the geophysical Observatory of Gibilmanna. The territory surveyed covers an area of approximately $3000 \mathrm{Sq}$. Kms.

The methods of survey, instruments and means used for the absolute and relative measurements carried out are described.

The processes for the elaboration of observation data are described and analyzed.

The regional anomalies of the covered area have been calculated through the difference from the values of the "normal field" for the components II and $\mathrm{Z}$ calculated with the observation data effectuated 
by the Italian Military Geografical Institute reduced to 1935,0 pertaining to 125 absolute stations.

The most outstanding anomalies are:

a) a positive anomalie of $\mathrm{Z}$ ( to $96 \gamma$ ) between Petralia and Gangi.

b) a negative anomalie of $\mathrm{H}($ to $-42 \gamma)$ in the area of Nicosia.

\section{BIBLIOGRAFIA}

(1) Giongi Mi., Medi E., Monelli C., Programma generale per una rele Geofisica Nazionale. Annali di Geofisica (in corso di stampa).

(2) Digifsi D., Sulla determinazione della componente orizzontale del c.m.t. e relativa carta italiana delle isodinamiche pubblicata dall'I.G.M. L'Universo, a. 23, n. $8,1942$.

Digiesi D.. Lu carta magnetica ditalia della componente orizzontale al 1940,0 editu dall I.G.M. Geofisica Pura e Applicata, v. IV, n. 2, 3, 1952.

(3) Istituto Geografico Militare, Carta magnetica dItalia alla scala 1:2.000.000 con le anomalie della declinazione e rappresentazione della componente orizzontale della perturbazione totale del c.m.t. al 1935,0.

Istituto Geografico Mijitare, Carta magnetica dItalia alla scala $1: 2.000 .000$ con le anomalie della componente orizzonitale al 1935,0.

Istituto Geografico Militare, Carta magnetica alla scala $1: 500.000$ della Sicilia al 1943,5 .

Istituto Geografico Militare, Carta magnetica d'Italia alla scala $1: 2.000 .000$ con le linee di uguale componente orizzontale al 1948.0.

(4) Monelu C., La rete geofisica e geodetica in Italia nel suo stato attuale $e$ nei suoi rapporti con la struttura geologica superficiale e profonda. I.N.G., Oss. Geofisico - Trieste, 1948.

(i) Chistoni M, Misure assolute degli elementi del magnetismo terrestre fatte in Sicilia. Annali dell'Uff. Centr. di Meteor. e Geod. v. XI, p. III, 1893 p. 371.

(c) Palazzo L., Risultati delle determinazioni magnetiche in Sicilia ecc. Rend. R. Acc. Lincei, v. VI, ren. 5, (331-337).

Palazzo L., Carte magnétique de la Sicilie. Terr. Magn., v. 4, 1899 187.92).

Palazzo L., Misure magnetiche fatte in Sicilia nel 1809. Annali dell Liff. Centr. di Meteor. e Geod. v. XVIII, parte I, 1896 (1-93).

(i) Petrucc, G., Notizie preliminari sullesplorazione regionale magnetica della Sicilia, L'Universo, a. XIII, n. 8, 1932.

(8) LA Cour, Le quartz-magnétomètre QHM, Publ. Det Danske Metcorologiske Institut, 1936.

(9) Heiland C. A., Geophysical exploration. New York, 1946.

(10) JoIce W., Manual on Geophysical prospecting with the magnetometer. U.S. Bureau of Mines, 1937.

(11) Bartels J., Veldkani J., Journal of Geophysical Research. V. 57, p. 416, 1952.

(19) НАцск H., Lehrbuck der angeuandten Geophysik. Berlino, 1931.

(13) Giongi M. Molina F., Campo normale e variazione secolare media degli elementi magnetici in Sicilia. Annali di Geofisica, VII, 1954. 


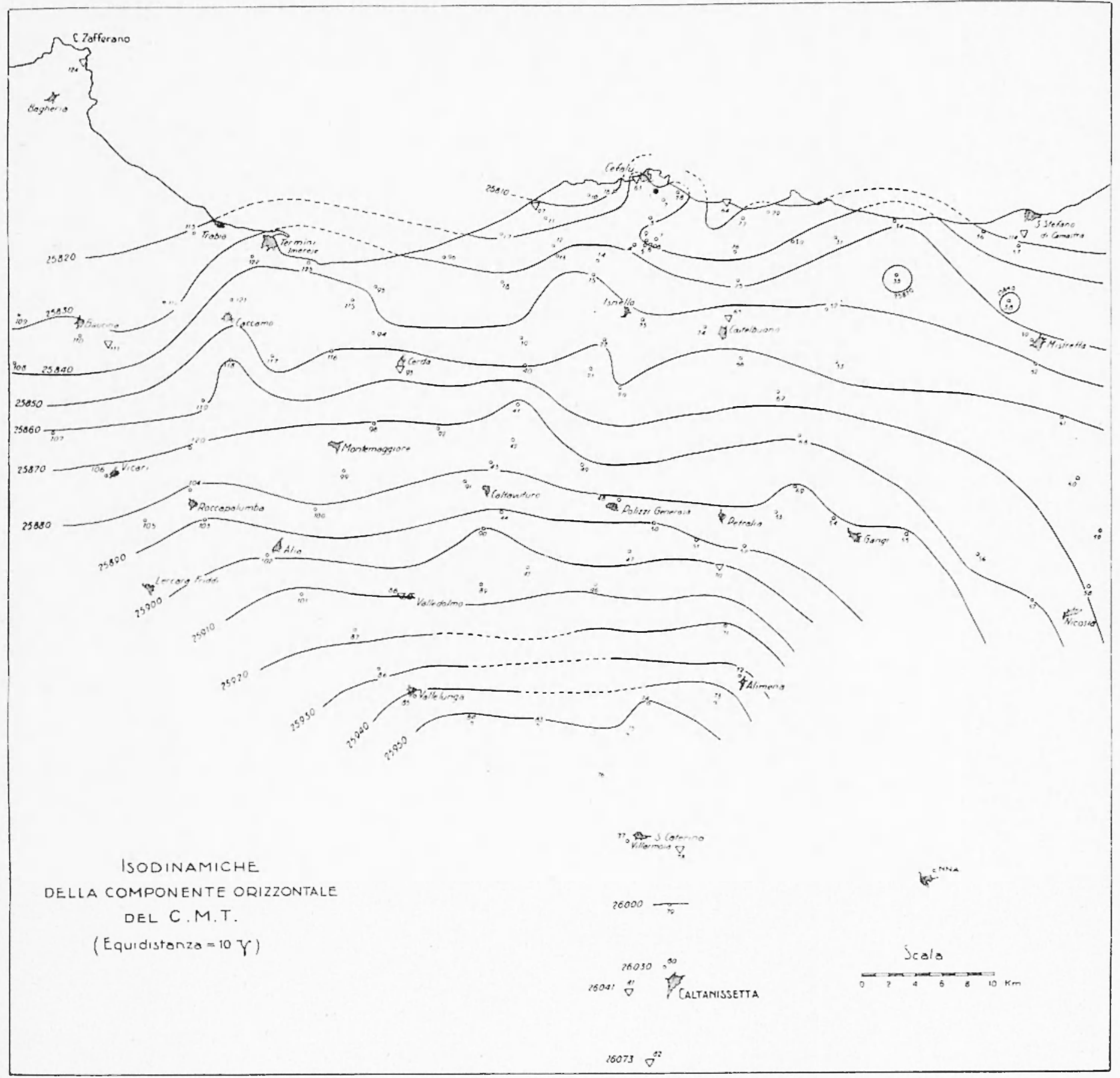

Fig. 3 


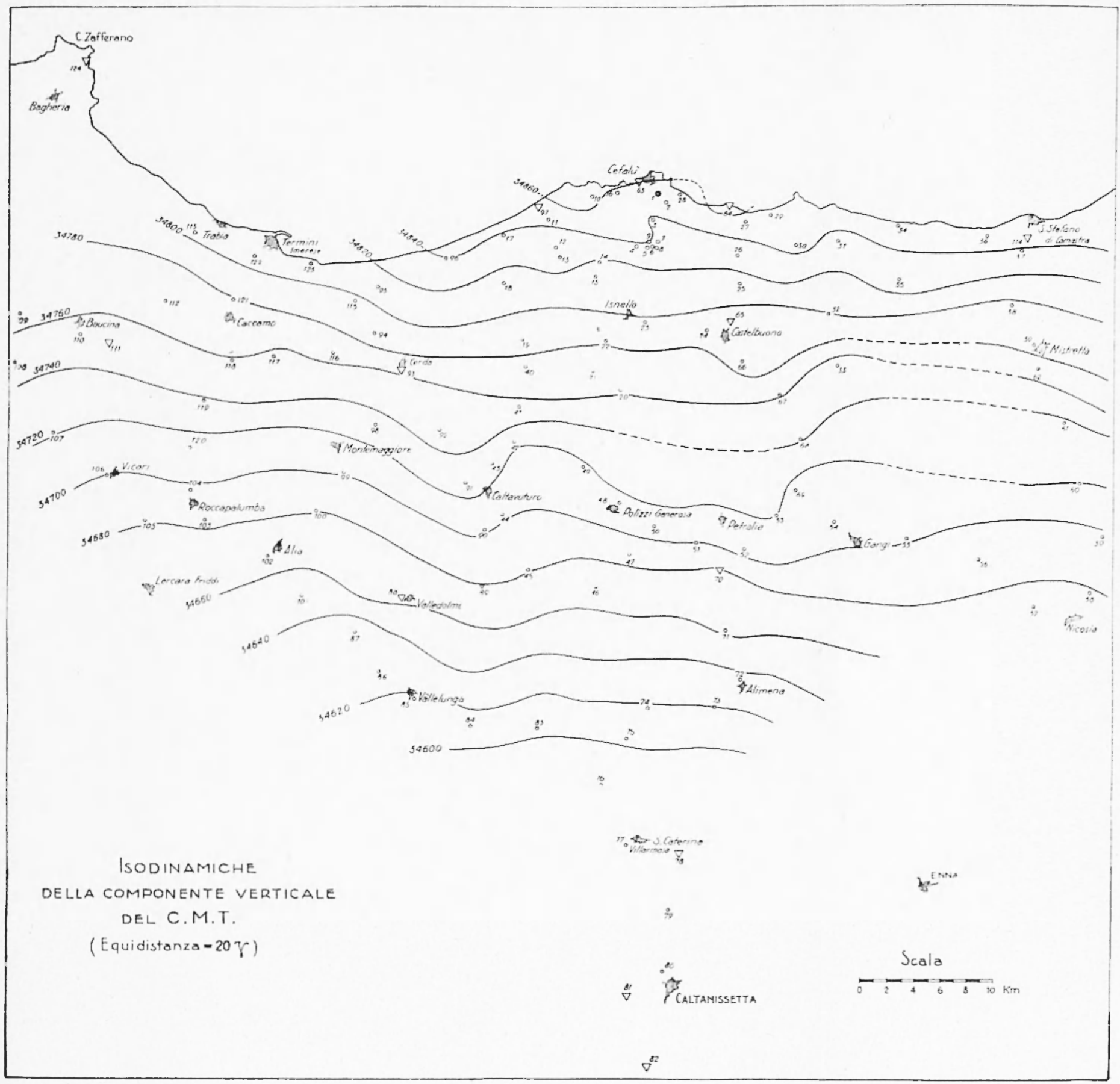

Iig. 1 


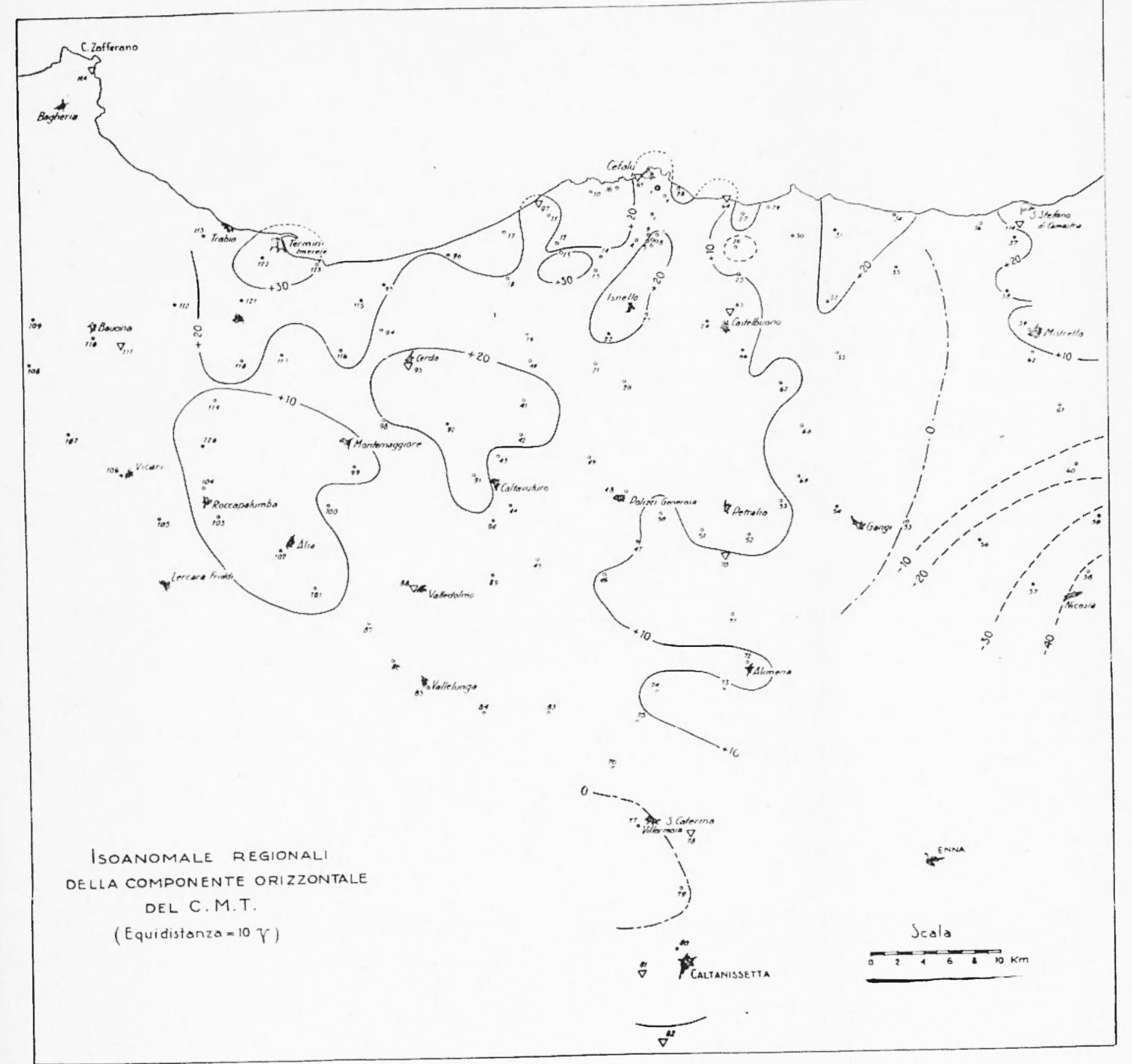

Fig. : 


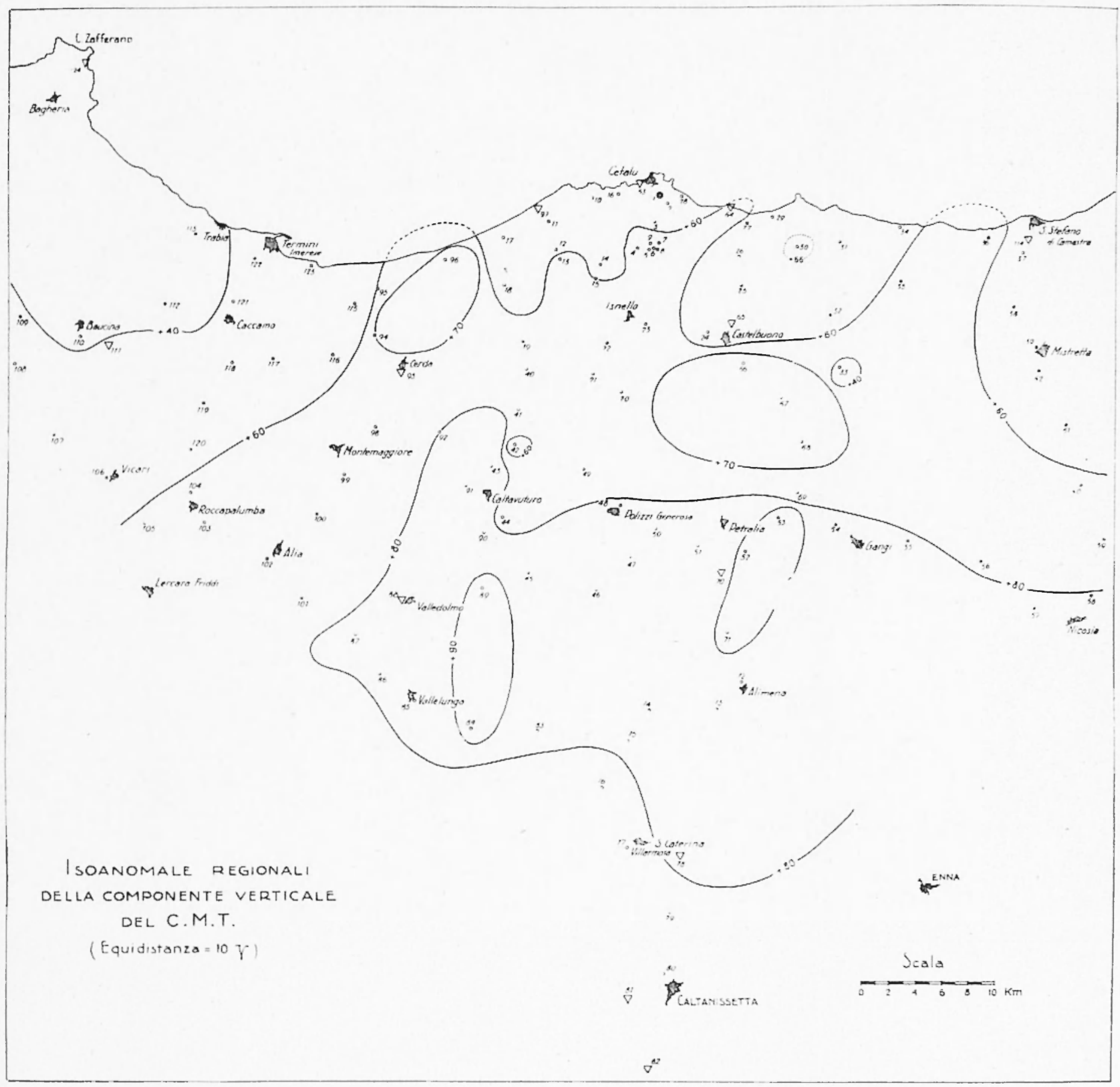

Fig. 6 